Check for updates

Cite this: RSC Adv., 2019, 9, 2055

\title{
Microfluidic assisted synthesis of PLGA drug delivery systems
}

\author{
Sima Rezvantalab and Mostafa Keshavarz Moraveji (D) *
}

Poly(lactic-co-glycolic acid) (PLGA) is a biocompatible and biodegradable polymer that recently attracted attention for use as part of drug delivery systems (DDS). In this context, there is an emerging need for a rapid, reliable and reproducible method of synthesis. Here, microfluidic systems provide great opportunities for synthesizing carriers in a tightly controlled manner and with low consumption of materials, energy and time. These miniature devices have been the focus of recent research since they can address the challenges inherent to the bulk system, e.g. low drug loading efficiency and encapsulation, broad size distribution and burst initial release. In this article, we provide an overview of current microfluidic systems used in drug delivery production, with a special focus on PLGA-based DDS. In this context, we highlight the advantages associated with the use of microchip systems in the fabrication of nanoparticles (NPs) and microparticles (MPs), e.g. in achieving complex morphologies. Furthermore, we discuss the challenges for selecting proper microfluidics for targeted DDS production in a translational setting and introduce strategies that are used to overcome microfluidics shortcomings, like low throughput for production.

Received 30th October 2018 Accepted 16th December 2018

DOI: $10.1039 / \mathrm{c} 8 \mathrm{ra0} 8972 \mathrm{~h}$

rsc.li/rsc-advances polymer with low water solubility and fast degradation rate under physiological conditions. On the contrary, poly(lactic acid) (PLA) is a stiff, hydrophobic polymer with low mechanical strength. As a copolymer, PLGA inherits the intrinsic properties of its constitutional monomers. PLGA properties can be tailored for specific applications just by varying the ratio between LA and GA monomers. Due to this phenomenon, PLGA represents a good material for drug delivery systems of many therapeutic agents (e.g. chemotherapeutics, as well as antiseptic, antiinflammatory and antioxidant drugs and proteins). Some of these PLGA based DDS have been approved by the US Food and Drug Administration (FDA) or are in clinical phase trials (Fig. 1).

Drug-loaded PLGA microparticles (MPs) ${ }^{6-8}$ and nanoparticles (NPs) ${ }^{9-12}$ can be synthesized via various bulk methods such as salting out, membrane emulsification, single/double-emulsion, nanoprecipitation. Particles produced by conventional bulk methods usually suffer from high batch-to-batch variation and polydispersity. These drawbacks arise due to uncontrollable synthesis method. ${ }^{\mathbf{1 3}}$ For example, all stages of NPs formation including nucleation, growth, and agglomeration take place simultaneously which leads to polydisperse particle formation. ${ }^{\mathbf{1 4}}$

Production process needs good control over surface charge, size and size distribution since these parameters control the drug release rate. In microfluidics the mixing rate, heat, and mass transfer are more precise, synthesis in these miniature devices is more controlled. Moreover, shorter mixing and process time provide another advantage of these small devices; as lower material consumption. These aspects of microfluidics are very advantageous comparing with conventional drug
Department of Chemical Engineering, Amirkabir University of Technology (Tehran Polytechnic), 424 Hafez Avenue, Tehran, 1591634311, Iran. E-mail: moraveji@aut. ac.ir 


\begin{tabular}{|c|c|c|c|}
\hline \multicolumn{2}{|c|}{ Clinicaltrials.gov identifier/ Phase NO. } & Brand name & Indication \\
\hline NCT03066245 & II & Somatuline®LA & Acromegaly \\
\hline NCT03060655 & II & Sandostatin ${ }^{\circledR}$ LAR & Acromegaly and carcinoid \\
\hline NCT02568527 & I & Nutropin Depot $(\mathbb{8}$ & Growth deficiency \\
\hline NCT03474900 & - & Zoladex & Breast cancer. Prostate cancer \\
\hline NCT02726646 & II & Arestin & Periodontal disease \\
\hline NCT01681381 & - & TrelstarTM Depot & Advanced Prostatic Cancer \\
\hline NCT01753089 & I & Suprecur ${ }^{(8)} \mathrm{MP}$ & Prostate cancer \\
\hline NCT03045913 & - & Pamorelin $^{(8)}$ & Prostate cancer \\
\hline NCT02578069 & - & Lupron Depot & Prostate cancer \\
\hline NCT03401216 & - & Eligard & Advanced Prostatic Cancer \\
\hline \multirow[t]{3}{*}{ NCT03429803 } & I \& II & Atridox & Chronic adult periodontitis \\
\hline & & Risperidal $^{(}$Consta & Antipsychotic \\
\hline & & Decapepty & Prostate cancer \\
\hline
\end{tabular}

Fig. 1 PLGA-based FDA-approved formulations ${ }^{15,16}$ and clinical trials.

production methods. Therefore these miniature devices can be used to address the conventional methods issues.

The aim of this review article is to collectively encompass the PLGA DDS produced in microfluidics, to address the impact of solvent and microfluidic system in the size and properties of PLGA-based drug delivery systems and pave the way for researchers to choose better system to achieve their goal in the production of drug delivery systems with sophisticated and precise properties.

\section{Microfluidics}

Microfluidics have been defined as devices in which small volumes (micro- or nanoliter) of liquids are processed or manipulated in microchannels to achieve better control on mixing, heat and/or mass transport. ${ }^{17}$ The microchannels are made of various materials such as polymers (e.g. polydimethylsiloxane (PDMS) or polyimide), metal (aluminum) and glass capillaries. As microfluidics synthesis allows to tightly control the properties of particles, the technology offers a broad range of advantages over the conventional bulk methods (Table 1). ${ }^{18-21}$ Microfluidics methods can be divided into two main categories based on the flow configuration in the microchannels: droplet-based (segmented) and continues microfluidics.

\subsection{Droplet-based microfluidics}

Droplet-based microfluidics are used to synthesize microdroplets, emulsions, and microparticles as well as

Table 1 The advantages and disadvantages of microfluidics for synthesis, 3,13,22,23

Advantages

- Tunable particle size

- Narrow size distribution

- Reproducibility

- The long duration of continues process

- More colloidal stability of the produced emulsion

- Well-controlled heat and mass transfer

- Large surface-to-volume ratio

- Low sample and solution consumption

\section{Disadvantages}

- PDMS is susceptible to solution

- PDMS is not resistant to high temperature \& pressure

- Difficult to online purification

- Difficult online characterization

- Microchannel clogging and fouling

- Limited production scale

- The high cost of glass, polytetrafluoroethylene

- Limited scale of aluminum microchannels 


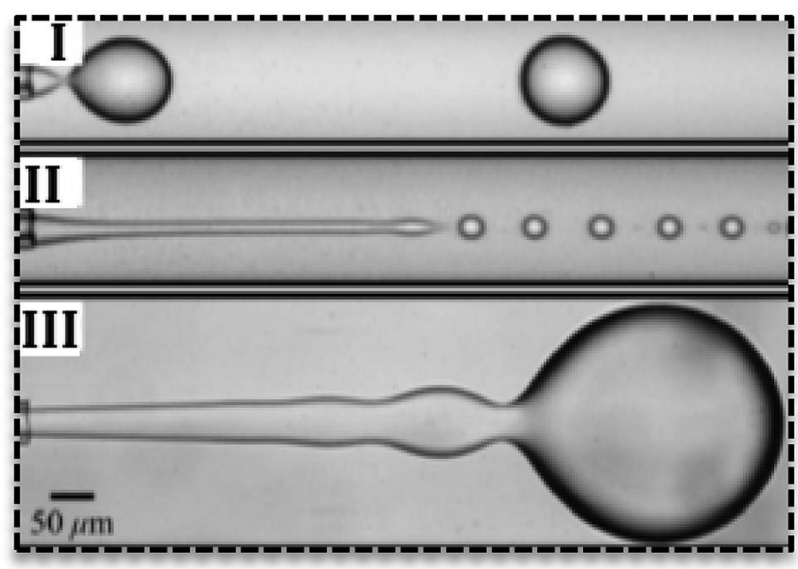

Fig. 2 Various flow regimes in the droplet-based microfluidics. (I) Dripping regime, (II) jetting regime, (III) squeezing regime in droplet generation. ${ }^{25}$

microreactors for nanomaterial production. In the current section, we will provide a brief description of the rules of thumb and principles of droplet generation.

Droplets form because of the instability in the inner flow which breaks into drops. Many parameters are important in the droplet formation, but crucial ones are channel geometry, flow rate, fluid viscosity and surfactant addition. For example, channel design (e.g. contraction or the method fluid flows come into contact) and channel diameter are determinant in the droplet formation phenomena and properties. Furthermore, fluid properties such as viscosities and the presence of surfactants are effective parameters in the viscous shear forces, which break the inner stream into droplets.

Droplets can form in various regimes of flow. ${ }^{24}$ Three main regimes are dripping, jetting and squeezing. The dripping mode has been observed in low flow rates and by an increase in the flow rate, it changes to jetting mode. ${ }^{25}$ Dripping regime produces droplets with narrow size distribution (Fig. 2I) while jetting mode produces polydisperse droplets. ${ }^{26}$ In the jetting regime, droplets are small with higher surface-to-volume ratio ${ }^{27}$ and form far from channels' exit (Fig. 2II).$^{25}$ In the squeezing mode (Fig. 2III), droplets start to grow and plug the continuous phase and consequently by the increase in the pressure of continuous phase they breaks off. Therefore, the squeezing mode is characterized by a fluctuation in the pressure of fluids.

Droplet generation as well as mixing in microfluidics can be performed by active and passive methods. ${ }^{24}$ In the active method, an external force (e.g. magnetic, electric and etc.) is applied to facilitate the droplet formation. In contrast, in the passive mode, two or more immiscible fluids come to contact in a junction and droplets form depending on the properties of the fluid (flow rate ratios, flow conditions, and the geometry of the device). ${ }^{25}$ Various geometries have been evaluated to promote droplet generation with single or multiple cores. ${ }^{27}$ Based on the flow contact, geometries are classified into three main categories: co-flow, cross-flow, and flow-focusing (Fig. 3I-III). ${ }^{28}$

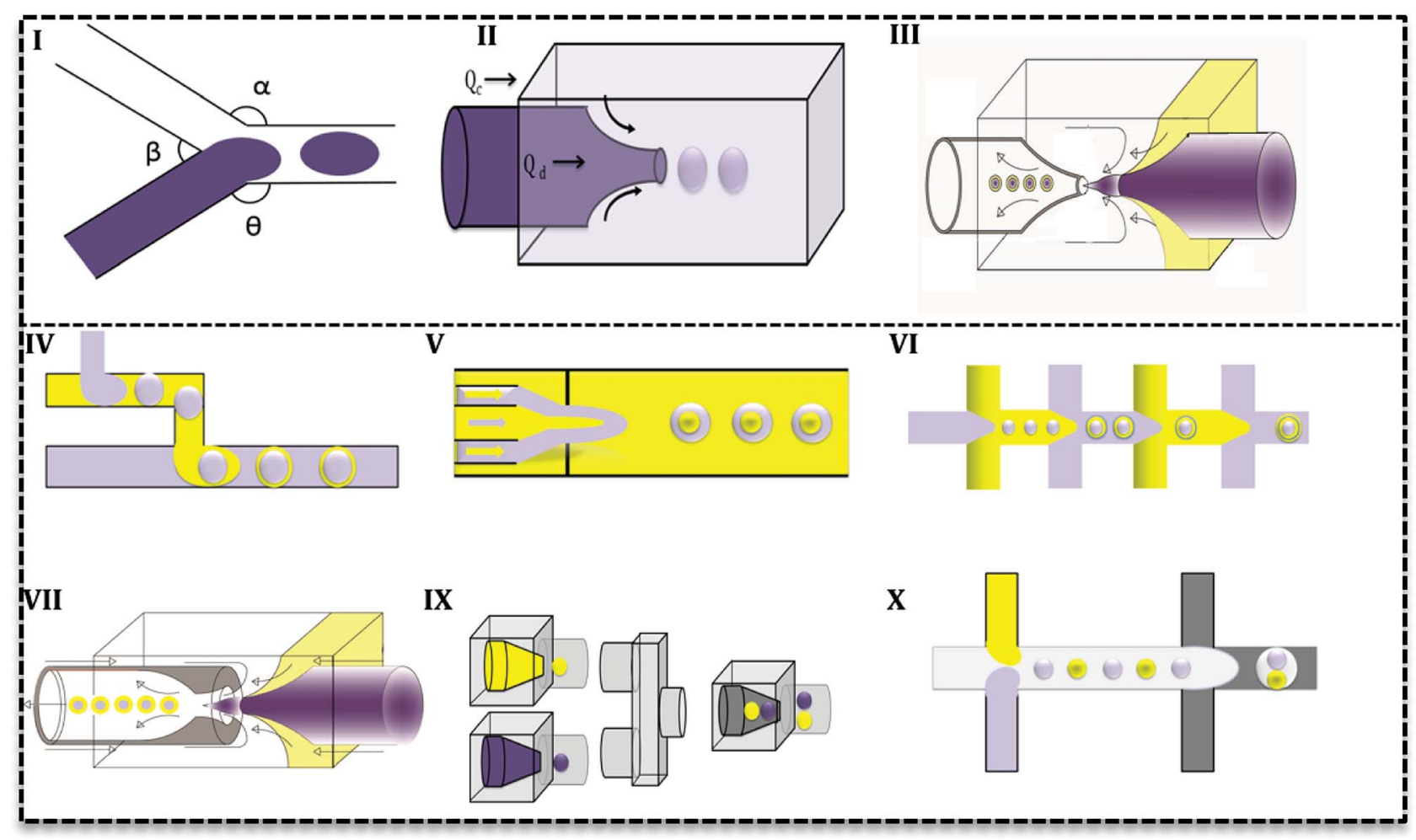

Fig. 3 Droplet-based microfluidic designs used to produce emulsions. (I) Cross-flow, (II) co-flow, (III) flow-focusing, (IV) two T-junctions in a row, (V) three concentric channels with one focusing point, (VI) flow-focusing in rows, (VII) combination of co-flow and flow-focusing geometries, (IX) three co-flow, (X) combination of two T-junction and flow-focusing. 
In cross-flow; continuous and dispersed phases meet in a junction with various angles (Fig. 3I) and both stream properties affect the flow and droplets form. Based on the angle of contact, there are various T-junctions $\left(\beta=180^{\circ}, \alpha=\Theta=90^{\circ}\right),(\Theta$ $\left.=180^{\circ}, \alpha>90^{\circ}, \beta<90^{\circ}\right)$ and $\left(\Theta=180^{\circ}, \alpha=\beta=90^{\circ}\right)$, Y-junction $(\beta$ $<90^{\circ}, 90^{\circ}<\alpha, \Theta<180^{\circ}$ ). Additionally, designs with more than two inlets have been used as double T-, V- and K-junction. ${ }^{25}$ Out of all junctions, the $\mathrm{T}$ - junction is most frequently used since it produces droplets with narrow size distribution. ${ }^{25,27}$ In a co-flow geometry, dispersed phase flows from an inner channel coaxially with the continuous phase in the outer channel with higher flow rate (Fig. 3II). Finally, in the flow-focusing method (Fig. 3III) two phases (dispersed and continuous) flow coaxially and pass through a region with contraction. Regarding the interfacial properties of the phases, at this point, the phase with lower flow rate breaks up into droplets and emulsion forms. Moreover, considering the number of flowing phases, it can produce single or double emulsions.

Aforementioned designs produce single emulsions. Double emulsion can be produced with a combination of them, such as T-junctions in cascade in glass capillary (Fig. 3IV), three co-axial channels with one contraction region (Fig. 3V), multiple flowfocusing designs in series (Fig. 3VI), and combination of a Tjunction and a flow-focus (Fig. 3VII). Many researchers have tried to produce multi emulsions in one step (Fig. 3VII), ${ }^{26,29}$ but it is difficult to control flow and the configuration becomes very complex. As it is seen in Fig. 3, microfluidics makes it possible and feasible to generate droplets with varying core size (Fig. 3IX and X) and one core with multiple shells (Fig. 3IX and X). Such droplet morphologies are the basis of microcapsules, core-shell MPs, polymersomes, lipid vesicles, etc. ${ }^{2}$

\subsection{Continuous phase flow microfluidics}

In this type, two or more fluids flow side-by-side in microchannels without segmentation or breakup. Researchers try to adopt continuous phase flow for material synthesis due to its reduced mixing time. ${ }^{30}$ A reduced mixing time, resulting from the compression by the outer fluid, is very important in many NPs syntheses since it provides a homogenous condition for NPs formation. As a result, NPs have a more narrow size distribution. Furthermore, uniform concentration, heat, and fluid profile take place in inner fluid and away from channel walls which prevent particle generation close to the channel wall and as a result, it reduces channel clogging. ${ }^{31}$

There are two types of devices for continuous phase flow microfluidics: the coaxial tube devices which are widely used for inorganic synthesis ${ }^{32-34}$ and the hydrodynamic focusing (HF) devices. The latter are very flexible in design and various subtypes have been recorded (Fig. 4II-IV) based on the number of fluids and angles of contact in the focus point. Fig. 4II shows the simplest HF design with one stream compressed between two streams in various contact angles $\left(\alpha \leq 90^{\circ}\right)$. Multiple HF is possible in a sequential manner (III) or one contraction point (IV). Depending on two angles $(\alpha \& \beta)$ and distances $\left(d \& d^{\prime}\right)$ this configuration can be used to improve flow stability or avoid central synthesis regime from channels wall. ${ }^{31}$ Recently, 3D designs of HF devices have been investigated in nanomaterial synthesis, in which the inner fluid squeezes between an outer flow horizontally and vertically. ${ }^{35-37}$ Coaxial tube designs (Fig. 4I) were also considered as 3D HF with circular cross section. ${ }^{27,38} 3 \mathrm{D}$ HF in microchannels with the rectangular crosssection is more complicated and difficult to achieve stable flow. Since in these 3D HF designs particle formation takes place away from channel walls, it prevents clogging and also aggregation of particles.

\subsection{Microfluidics systems used in PLGA drug delivery systems}

In the last two decades, PLGA-based drug delivery systems are being produced in microfluidics. There are several reports of

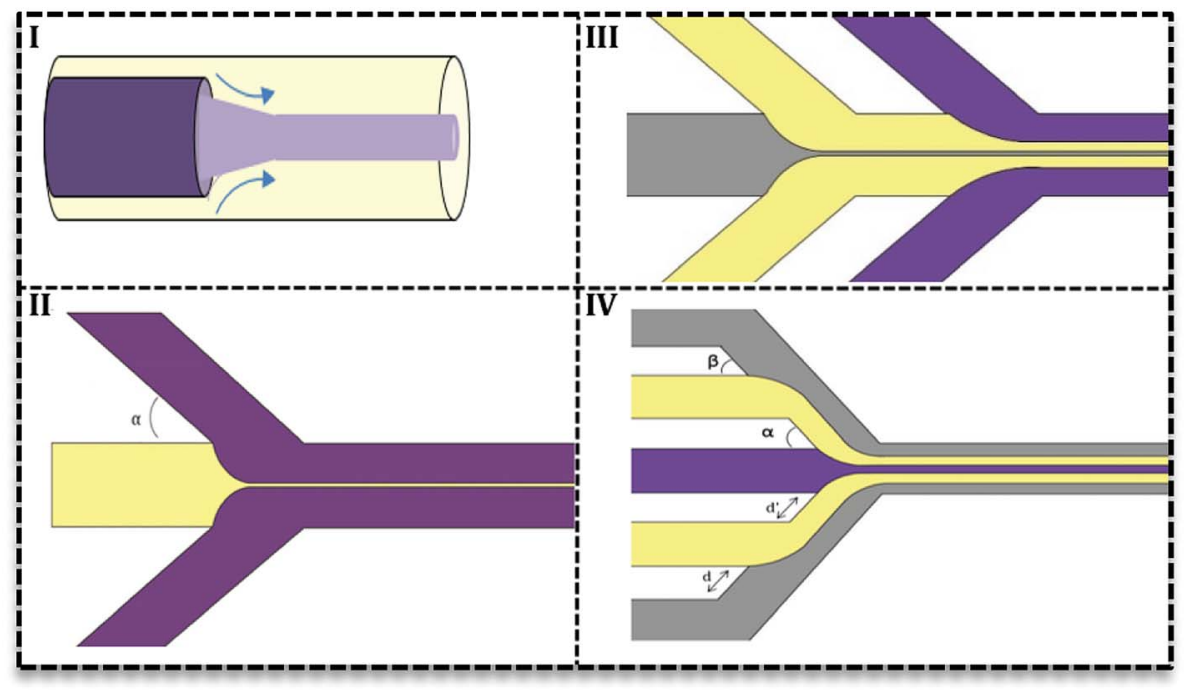

Fig. 4 Hydrodynamic Focusing (HF) designs in continuous microfluidics. (I) Co-axial devices, (II) simple HF design in which the central flow is squeezed by two sheath flows from two sides with various angles. (III) HF design with additional sheath-flows to improve flow stability, (IV) multiple HF steps. 
PLGA-based MPs, NPs and microfibers produced using this technology. A wide range of drugs has been loaded into PLGAbased MPs and NPs (e.g., bupivacaine, ${ }^{39}$ risperidone, ${ }^{40}$ ibuprofen, ${ }^{41}$ paclitaxel (PTX), ${ }^{42}$ doxorubicin (DOX), ${ }^{43}$ camptothecin ${ }^{43}$ and etc.) in various microchannels such as PDMS microfluidics, ${ }^{39}$ glass capillary, ${ }^{43}$ phenol formaldehyde resinbased microfluidic chip, ${ }^{44}$ aluminum ${ }^{22}$ and silicon. ${ }^{45}$ However, production of microfibers in the microfluidics is not the commonly used synthesis method, especially for drug delivery purposes. The method has been employed to produce microfibers for tissue engineering scaffolds. ${ }^{46,47}$ However, microfibers produced by this method have shortcomings to be used in DDS such as the presence of voids in the microfibers structure ${ }^{\mathbf{4 8 , 4 9}}$ and hydrogel nature of the produced fibers due to solidification process. ${ }^{50}$

Considering the microfluidics configuration, various methods of flow and mixing have been employed for NPs production in micro-channels such as segmented or continuous phase flow. Inorganic nanomaterials are synthesized in segmented flow or droplets based microfluidics. ${ }^{51-57}$ In such systems, micro-scale droplets serve as micro-reactors. In the case of PLGA, use of droplet-based microfluidics results into micron size particles. MPs have been synthesized via reactions or phenomena that turn emulsions and droplet templates into particles. These phenomena include solvent evaporation or extraction which is not easily achievable in nano-scale.
Furthermore, according to the reports, droplet sizes are proportional to the size of the channels. Nano-scale droplets are necessary for NPs production. On the other hand, manipulation and pumping of fluids in such small size channels and capillaries need a large amount of power. Additionally, it is almost impossible to online characterize and control the fluids and droplets properties in nano-scale.

Literature survey led us to the idea represented in Fig. 5, i.e. the use of droplet-based microfluidics produces PLGA MPs and particle synthesis in the continuous microfluidics will end up at nano-scale. In Fig. 5 each line with a number over it indicates the size range reported by the associated reference article. It can be seen that the type of microfluidics is very important in the scale of the drug delivery system. In more detail, except a few papers, there are not many reports on the PLGA nanoparticles production via droplet microfluidics. ${ }^{26}$ For instance, Lee et al. succeeded in PLGA micro/nanosphere production through droplet-based microfluidics. They investigated solvent evaporation and extraction effect in the final particles sizes. Fast evaporation of the PLGA droplets in dimethyl carbonate (DMC) produced PLGA MPs (3 to $30 \mu \mathrm{m}) .{ }^{58}$ However, they generated PLGA droplets in dimethyl sulfoxide (DMSO) in silicon oil continuous phase and after infusion with water droplets, solvent extraction led to nano-size (70 to $500 \mathrm{~nm}$ ) PLGA particle formation. In both methods, particle size increases with the higher concentration of PLGA. In another report, Janus PLGA

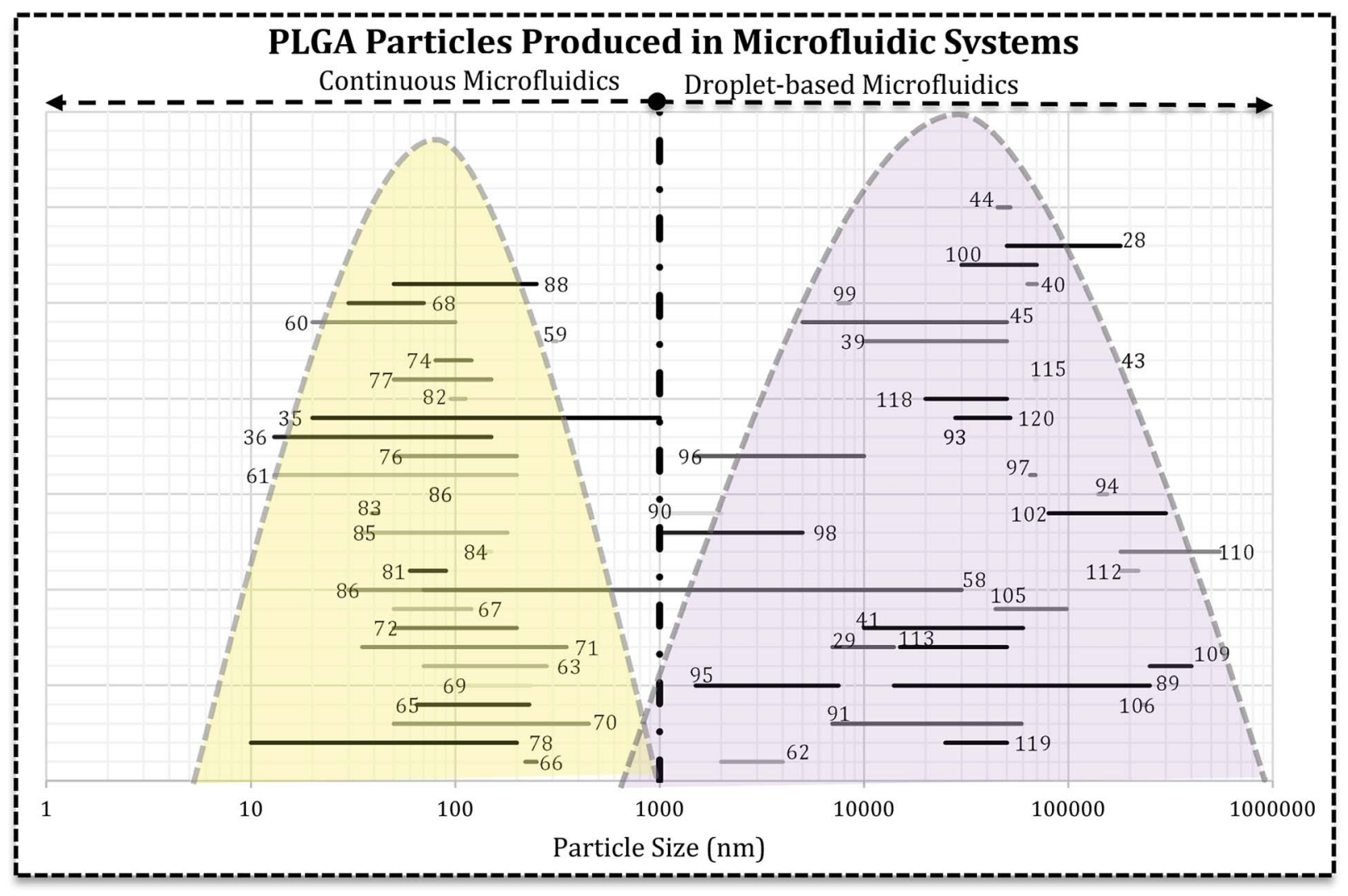

Fig. 5 The relationship between microfluidics type and the final size of PLGA-based drug delivery systems. Each line represents the size range of DDS reported in the related reference that is produced in droplet-based or continuous microfluidics. The reader can refer to desired section (e.g. Janus MPs Section 4.2) and the references numbers (ref. 89, 116-120) and find the related lines. 
NPs have been synthesized with T-junction fluidic device consisted of two stainless steel capillaries that enter in a transparent plastic tube. From one inlet PLGA/PLA and Nile red mixture in dimethylformamide (DMF) and the other PLGA and rhodamine $6 \mathrm{G}$ in acetone added (with $100 \mu \mathrm{L} \mathrm{h}^{-1}$ flow rate) to an aqueous solution containing 1\% PVA (flow rate 10 $\mathrm{mL} \min ^{-1}$ ). The authors used the same system to load PTX and DOX in PLGA NPs and claimed that the approach overcomes both drugs limitations and offers a high yield of dual-loaded NPS. ${ }^{59}$

Fig. 5 reveals that directly the microfluidic type and indirectly, solvent miscibility, flow rates, and their ratio, the synthesis processes are very crucial in the final characteristics of the drug delivery system and efficiency. It appears that the particle formation process is critical in the final size and size distribution of the carrier. For example, PLGA-PEG solution in a water miscible solvent (acetonitrile, DMSO, DMF) focused with aqueous solution gives rise to self-assembly of polymer chains into polymeric NPs and micelles. ${ }^{\mathbf{1 4 , 6 0 , 6 1}}$ However, the similar polymeric composition in a water immiscible or semimiscible solvent (DCM) produces droplets which need a further step to remove the solvent and produce MPs. ${ }^{62}$ For example, change in the solvent from DCM to DMSO and also infusion with water droplets resulted in the sub-micron particles. Although they claimed the droplet-based microfluidic produced nano-scale PLGA particles, the infusion of polymer mixture (water-miscible solvent) with water droplets produced NPs which the precipitation takes place within the droplets. ${ }^{58}$ Additionally, NPs fabricated using DMF as an organic solvent in the T-junction droplet producing a fluidic chip with very low flow ratio. ${ }^{59}$ It can be concluded that precursor's properties and the configuration of streams in microchannels have the decisive influence in the formation process. Although the device resembles droplet-based microfluidics, comparison of dispersed and continues flow rates brings dropwise nanoprecipitation (in the bulk method) into mind. Moreover, for NPs production solvent can significantly affect the properties of drug loaded systems (size and size distribution). ${ }^{\mathbf{6 3 , 6 4}}$ Solvents with higher diffusion coefficient produce NPs with smaller size and narrower size distribution. ${ }^{64}$

PLGA NPs produced in continuous microfluidic are in submicron size with low encapsulation efficiency and drug loading. This challenge is associated with water miscibility of the solvents. During the NPs formation, as a result of solvent displacement, polymer chain come together and form smaller particles. However, a huge part of agents is lost at the same time during solvent displacement. Recently, in an interesting approach, $\mathrm{Xu}$ et al. used glass capillary droplet-based microfluidics in which emulsion was generated by the combination of two solvents; DMSO and DCM. ${ }^{65}$ Although the organic phase forms droplets, one of the solvents (DMSO) displaces into aqueous phase while DCM entraps the drug (DOX) within the droplets during NPs formation, avoiding drug lost. In agreement with the statement, encapsulation efficiency $(48.5,49.9$, and $56.9 \%)$ and drug loading $(9.7,10.0$, and $11.4 \%)$ increase in NPs with increase in the DCM ratio in solvent mixture $\left(V_{\mathrm{DCM}}\right)$ $V_{\text {DMSO: }} 0,0.05,0.1$ ).
Taken together, the particle formation process is the basic determining factor of the DDS size. In the continuous microfluidics, PLGA particles form by a nanoprecipitation process in the interface of water-miscible organic solution (middle stream) with the aqueous stream from both side and even sometimes from up and downside. This process is very fast and happens in nano-scale and eventually NPs form. However, in the case of droplet microfluidics, PLGA polymer with drug or agent dissolved in a water-immiscible (or partially miscible) solvent produce template emulsion or droplets that typically evaporated to remove the solvent and produce MPs. As a result, the organic phase solvent and microfluidics configuration regulate the particle formation process, pace and consequently the produced particle size.

\section{PLGA-based nanoparticles}

As it has been mentioned in the previous section, PLGA NPs synthesis in continuous flow microfluidics is accomplished by the nanoprecipitation process between two phases that flow alongside. In this process, a material solution containing polymer and drugs in a water-miscible solvent (e.g. acetone, acetonitrile, ethanol, or methanol) is compressed within a nonsolvent phase such as an aqueous solution containing surfactants. ${ }^{66}$ The solvent is miscible in non-solvent and transfers between two phases which leads to NPs formation. ${ }^{30}$ Various kind of microfluidics (2D HF to $3 \mathrm{D}$, laminar flow to turbulent jet) has been used to produce PLGA-based NPs.

The simple yet convenient 2D HF type allows fabrication of multi-drug loaded PLGA NPs with desired properties. For example, bisphosphonate conjugated PLGA (BP-PLGA) NPs loaded with superparamagnetic iron oxide nanoparticles (SPIONs) and PTX are produced in a PDMS based chip (Fig. 6I) to be used in chemotherapy, hyperthermia, and MRI diagnosis. ${ }^{67}$ Results show that NPs produced in microfluidics (in the range of 40 to $100 \mathrm{~nm}$ ) is smaller in comparison with bulk method ( $\geq 120 \mathrm{~nm}$ ). Furthermore, it is possible to control the properties of NPs with flow conditions in 2D HF microfluidics. For example, by an increase in the flow rate ratio (the ratio of the organic phase containing NPs precursors to aqueous phase), final NPs size increases (Fig. 6II). Additionally, this ratio can affect drug release profile, as in lower ratios, NPs are smaller and more compact, consequently, drug release is slower. Results from in vivo analysis with a bone metastasis model (MDA-MB-231) mice showed that tumor growth suppression and apoptosis level enhanced with targeted microfluidic NPs. In a recent study, ${ }^{68}$ it was shown that an increase in the flow ratio from 0.025 to 0.125 means size of non-targeted curcuminloaded PLGA NPs increase from about $30 \mathrm{~nm}$ up to $70 \mathrm{~nm}$. The authors stated that the higher the ratio, the broader the size distribution of produced NPs. Results from in vitro analysis exhibited that compared with free curcumin, microfluidically produced NPs enhance the antitumor activity of the drug toward leukemia Jurkat cells. As it's seen, flow ratio is an effective parameter in the synthesis process and lower flow ratio produces smaller NPs with narrow size distribution. However, lower flow ratio means the slower flow of NPs precursors and an 


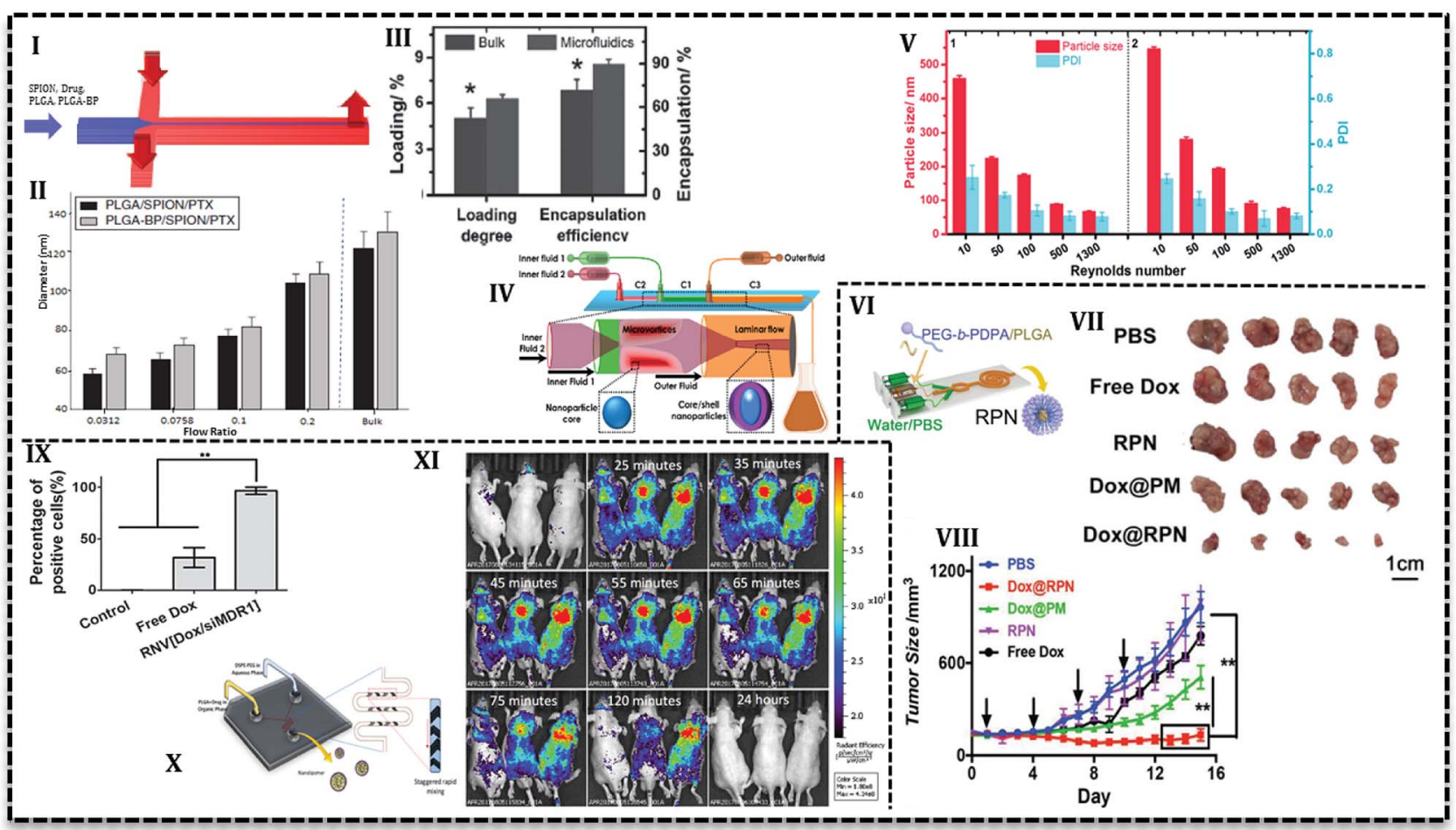

Fig. 6 PLGA-based NPs produced in continuous microfluidics. (I) Schematic of 2D HF microfluidics used to load SPION and PTX in BP-PLGA NPs. (II) Diagram of NPs size as a function of flow ratio in the microchannels. NPs size grows with the rise in flow ratios. (III) Drug loading and encapsulation efficiency are higher for NPs prepared by microfluidics compared with the bulk method. (IV) Schematic of two sequential glass capillaries used for the preparation of core-shell NPs with homogeneous size distribution through rapid mixing. (V) NPs size and polydispersity index drop significantly in higher Reynolds numbers. (VI) The polymeric solution consisted of drug and PEG- $b$-PDPA/PLGA in DMF and trifluoroethanol (TFE) is focused from two sides with an aqueous solution containing PBS in 2D HF microfluidics. (VII) Images of ex vivo tumors treated with PBS, free DOX, and rigid pH sensitive NPs (RPN), DOX-loaded micelles without PLGA core (DOX(APM), and DOX-loaded RPN (DOX@RPN). (VIII) Tumor volume treated PBS, free DOX, and RPN, DOX@PM, and DOX@RPN indicate that core/shell NPs with PLGA core have higher antitumor activities among other groups. (IX) Co-incubation of free DOX and siMDR1 and Dox-loaded NPs with MCF-7/ADR cells for $2 \mathrm{~h}$ show that NPs are completely (100\%) taken up while free DOX cellular uptake is $30 \%$. (X) Micromixer with a herringbone pattern to improve mixing efficiency used for curcumin-loaded PLGA-lipid NPs. (XI) In vivo evaluation with C4-2B xenograft mice shows that after 24 hours is removed completely from the body.

undesirable consequence of slow flow is low efficiency which is the intrinsic characteristic of low flow rates.

Another effective parameter is mixing time and determined by the design of the microfluidic chip. Mixing time controls the particle formation and also final properties and the amount of NPs. In 2D HF microfluidics with microchannels, flows are laminar; mixing is based on diffusion which takes place in the interface of phases. In order to investigate the effect of design on mixing time and also NPs properties, PLGADOX solution in the mixture of DMF and trifluoroethanol (TFE) was introduced into three PDMS based chips: 2D flat HF, 3D arc, and 3D double spiral. ${ }^{69}$ Simulation results represented that the mixing time as a function of flow rate can be prolonged by an increase in flow rate. Moreover, 3D designs have a shorter mixing time due to the shortened mixing distance. For instance, in the same flow rate $\left(2.5 \mathrm{~mL} \mathrm{~h}^{-1}\right)$ mixing time decrease for 2D flat HF, 3D arc and 3D double spiral $(29,16$, and $14.5 \mathrm{~ms}$, respectively). However, encapsulation efficiency (ca. 50\%) and cellular uptake with MCF-7 and HeLa cells are reported only for $100 \mathrm{~nm}$ NPs produced in origami chip. DOXloaded PLGA NPs were more taken up with cancer cells and showed higher cytotoxicity compared with free DOX. In another conducted research, Liu et al. used 3D coaxial flow in the capillary glass to minimize the mixing time. They increased the flow rates which caused flow regime transfer from laminar to turbulent jet. ${ }^{63}$ An additional distinguishing feature of their design is that organic phase (PLGA solution and PTX) flows as outer fluid near the wall of the channel and aqueous phase flows in the central glass capillary. Higher drug loading and encapsulation efficiency (Fig. 6III) reported for microfluidic NPs compared with the bulk method. The results attributed to the fact that volume ratio between polymer precursor and an aqueous solution for the microfluidics system is fixed and higher than bulk synthesis (the ratio is low and increases gradually during the process). PLGA NPs with the size ranges of $100-210 \mathrm{~nm}$ can be produced with mass production rates up to $242.8 \mathrm{~g}$ per day. In a similar configuration with one more capillary glass (Fig. 6IV) PTX and sorafenib (SFN) (anti-angiogenic drug) are assessed to be loaded in core-shell NPs. ${ }^{70}$ Results declare that drug loading increases for PTX (from 6.7\% to $42.6 \%$ ) and SFN (from $6.2 \%$ to $45.2 \%)$ with a sequential configuration in comparison with single step process. As it's seen in Fig. 6V, the higher the flow rates (higher Reynolds number) the smaller the NPs size. 
Microchannel size is another effective parameter in the formation and properties of NPs. In larger diameter channels the ratio drops and NPs size increases. The smaller size of the NPs produced in microfluidics is a result of a higher surface-tovolume ratio offered by these devices. In a 3D microfluidics fabricated out of commercially stainless steel capillary with three inlets, dexamethasone and ribavirin encapsulated in NPs with size range 35-350 and 50-200, respectively. ${ }^{71,72}$ According to the outcomes, by a decrease in the internal diameter of the channel from 600 to $130 \mu \mathrm{m}$, NPs size dropped from about 133 to $28 \mathrm{~nm}$. Researchers mentioned that easily assembled device has the capability to be used in a series of parallel designs for mass production up to $2.4 \mathrm{~kg}$ NPs per day. ${ }^{71}$

\subsection{PEG-PLGA NPS}

PEGylation is the process of polyethylene glycol (PEG) chains conjugation on the molecules or microstructures. The NPs consists of PLGA core and PEG chains on the surface offer a wide range of advantages; e.g. long circulation time due to immune system evade, small size around 20-100 nm, higher solubility, and stability, capability for drug encapsulation, good degradability, and biocompatibility. ${ }^{73}$ PEG chain conjugation to PLGA has been investigated for a long time. PLGA-PEG copolymers assemble into NPs or micelles in the aqueous phase. Therefore, the precipitation in the aqueous phase to produce NPs is being used for a long time in the bulk method and it has been adapted to the microfluidic system in the last decade.

For the first time, Karnik et al. reported nanoprecipitation of PLGA $_{15 \mathrm{k}}-\mathrm{PEG}_{4 \mathrm{k}}$ in $2 \mathrm{D}$ HF microfluidics. ${ }^{\mathbf{1 4}}$ They reported that NPs size and drug encapsulation efficiency are affected by flow rates and composition of phases, i.e. incorporation of PLGA increases encapsulation efficiency from $28 \%$ to $51 \%$. Incorporation of PLGA or PLA increases the NPs size in bulk method. However, to prevent the size enlargement, PLA modified with prodrug (platinum(Iv) [Pt(Iv)]) has been incorporated to PLGAPEG for drug release control. ${ }^{74}$ The polymer solution used to load hydrophilic cisplatin and hydrophobic docetaxel into NPs in a 2D HF microfluidic. Nanoprecipitation with identical condition produced smaller NPs in a microfluidics $(\sim 100 \mathrm{~nm})$ compared with the bulk method (greater than $150 \mathrm{~nm}$ ). In a similar study, cisplatin prodrug (as conjugated to PLA backbone) and free irinotecan loaded into PLGA-PEG NPs to target prostate-specific membrane antigen (PSMA) overexpressing prostate cancer cell using $S, S$-2-(3-[5-amino-1-carboxypentyl]ureido)-pentanedioic acid ligand. ${ }^{60}$ Addition of cisplatin-PLA to the solution of PLGA-PEG with irinotecan increased its encapsulation efficiency from $10 \%$ up to $44 \%$. For both studies, results from in vitro analysis with LNCaP cells showed that the combination of drugs increases the cytotoxicity toward the cells compared with single drug-loaded NPs. In the following study, the authors used two-stage microfluidics for mixing and production of doxorubicin-loaded PLGA-PEG targeted NPs in a fully integrated microfluidic device. ${ }^{61}$ They could produce a library of NPs with various surface properties, ligand densities, size, and molecular weight to evaluate in vitro and in vivo. Addition of $14 \%$ mole of targeted polymer to PLGA-PEG mixture increases the LNCaP cells uptake and tumor accumulate up to 3.5-fold compared with bare PLGA-PEG NPs. In order to promote the cellular uptake, $\mathrm{pH}$-sensitive NPs have been produced in a similar 2D HF microfluidics (Fig. 6VI) which can escape endo/lysosomes and overcome drug resistance. ${ }^{75}$ The core-shell NPs consisted of DOX-loaded PLGA core and poly(ethylene glycol)-poly(2-(diisopropylamino)ethyl methacrylate) (PEG- $b$-PDPA) diblock copolymer shell. The authors claimed that upon the translocation in the acidic endocytic, the residual parts of shell produce positive charges over the PLGA core and help lysosomal escape. Results for ex vivo and in vivo analysis with MCF-7/ADR tumor-bearing mice reveal that in comparison with free DOX, the NPs significantly suppress the drug-resistant tumor growth (Fig. 6VII and VIII).

To produce as small as possible NPs with homogeneous size, PLGA-PEG self-assembly investigated in 3D hydrodynamic focusing microfluidics with sequential inlets. ${ }^{35,36}$ Results disclosed that not only the concentration of polymer is important but also polymer molecular weight is a contributing factor in the final size of NPs. For instance, with an increase in PLGA molecular weight (10 to $90 \mathrm{kDa}$ ) the produced NPs size increases ( $\sim 26$ to $150 \mathrm{~nm}$ ). Moreover, in the same molecular weight (10 $\mathrm{kDa})$ with increase in the concentration (10 to $50 \mathrm{mg} \mathrm{mL}^{-1}$ ) the NPs size increases (13 to $26 \mathrm{~nm}$, respectively). The 3D microfluidics has reduced the fouling and clogging since reaction takes place far away channels wall. ${ }^{35}$ However, the $3 \mathrm{D}$ microfluidics is complicated and it is difficult to achieve stable flow and reproducible manner. To increase the throughput, 8 parallel 3D devices in one chip, they reduced the batch time (for $25 \mathrm{mg}$ ) from $5 \mathrm{~h}$ to less than 20 minutes. $^{36}$

Low throughput of PEGylated PLGA NPs produced by these miniature devices is an important challenge and it has been tried to overcome in various research. In one attempt a 3 layer PDMS microfluidics with 100 channels fabricated that produced methoxyl PEG-PLGA (MPEG-PLGA) up to $0.5-2.0 \mathrm{~mL} \mathrm{~h}^{-1}$ polymer flow rate with narrow size distribution. ${ }^{76}$ Another $3 \mathrm{D} \mathrm{HF}$ microfluidics fabricated with parallel polyimide films which can tolerate up to $16 \mathrm{MPa}$ with high throughput up to $331 \mathrm{~g}$ per day of PEG-PLGA NPs. ${ }^{77}$ Lim et al. ${ }^{78}$ designed a turbulent jet micromixer with a higher flow rate and consequently high production more than $3 \mathrm{~kg}$ per day which is the highest throughput achieved up until now. ${ }^{3}$

\subsection{Lipid-PLGA NPS}

Lipids are hydrophobic or amphiphilic molecules which also can be used to modify molecules. They have attracted attention for PLGA surface modification. ${ }^{79}$ These core/shell structures have hydrophobic cores and hydrophilic tails of lipids. These structures are capable of hydrophobic drug loading and have prolonged circulation time compared to PLGA NPs. ${ }^{\mathbf{7 9 , 8 0}}$

Recently, lipid-PLGA NPs have been produced in the microfluidic device to control the reaction and properties of the particles. In agreement with results, lipid-PLGA NPs with a smaller size $(\sim 62.5$ and $\sim 87 \mathrm{~nm})$ are produced in lower total flow rate $\left(41,246 \mathrm{~mL} \mathrm{~h}^{-1}\right.$, respectively). Moreover, cellular uptake evaluation with A375 cells (human melanoma cell line) 
indicated that smaller lipid-PLGA NPs are internalized more efficiently compared with larger counterparts. ${ }^{81}$ Zhang et al. synthesized dual drug (DOX and combretastatin A4 (CA4)) loaded PLGA NPs with mono- and bi-layer lipid shells in a twostage HF microfluidics. ${ }^{82}$ Cellular uptake analysis with HeLa (cervical cancer cells) and HUVEC cells (Human umbilical vein endothelial cells) showed that NPs with monolayer lipid was taken up more than bilayer counterparts and even free drugs. Furthermore, similar findings observed with in vivo and ex vivo analysis, i.e. monolayer NPs exhibited improved anticancer activity and faster tumor accumulation. The same procedure adapted to produce lipid-PLGA and lipid-water-PLGA NPs loaded with doxorubicin in PLGA core and CA4 in the shell, respectively. Rigidity analysis showed that NPs with a layer of water between polymer and lipid layers are more flexible than the bi-layer counterparts. In vitro analysis (with HeLa and HUVEC cells) verified the results from a molecular dynamics simulation that revealed rigid NPs have enhanced cellular uptake compared with free drugs and flexible NPs. ${ }^{83}$ According to the findings that rigid lipid-PLGA NPs exhibit higher cellular uptake, similar morphology adapted to load hydrophilic agent (siRNA) in water core, hydrophobic drug (DOX) in PLGA layer and lipid shell. The core/shell morphology enabled co-delivery of siMDR1 (the siRNA sequence against the multi-drug resistant protein) and doxorubicin and performed an enhanced gene knockdown efficiency compared with lipofectamine ${ }^{2000}$. In vitro analysis (Fig. 6IX) by MCF-7/ADR cells showed that cellular uptake of the lipid-PLGA NPs $(\sim 100 \%)$ is much higher than free DOX (30\%). The NPs was surprisingly effective in tumor growth inhibition for mice treatment in comparison with and free drug, free gene. ${ }^{84}$

Lipid shells not only used to load drugs also improved the stability of polymeric NPs but also used to enhance quantum dot (QD) nanocrystals hydrophilicity and biocompatibility in biological environments. In a conducted study, PLGA solution in acetonitrile focused in a 2D HF microfluidics with lipid solution (aqueous solution of lecithin and 1,2-distearoyl-snglycero-3-phosphoethanolamine- $\mathrm{N}$-[carboxy(polyethylene

glycol)] (DSPE-PEG)) and afterward mixed in a Tesla microstructure to produce lipid-PLGA NPs. Additionally, in order to load quantum dots for diagnosis application, they used the same arrangement and solutions in which the aqueous phase contained lipophilic quantum dots (dissolved in tetrahydrofuran) ${ }^{85}$ Results exhibited that rapid mixing in Tesla micromixers produces monodisperse NPs $(35-180 \mathrm{~nm})$ since it improves mixing efficiency. Results with various experiment revealed that lipid-PLGA NPs with $40 \mathrm{~nm}$ size have the most stable form and also lipid : PLGA (e.g. $1: 10$ to $1: 1000)$ ratio is not effective in the size of NPs. Another imaging agent (gold nanocrystals (AuNCs)) with two therapeutic loaded in lipidPLGA NPs. With an interesting approach, PLGA which was functionalized with AuNCs forms a hydrophobic core loaded with DOX and a lipid layer contains SRF, lipid shell composed of ordinary phospholipids and PEGylated phospholipids. ${ }^{86}$ Another interesting point about the study is the use of a 3D microfluidic chip with three inlets to increase the production and control the size of NPs. Drug release analysis reveals a sequential release of drugs; SRF release followed by DOX release and in vivo evaluations represent higher accumulation in the tumor site. Kim et al. ${ }^{87}$ used the same chip to investigate the flow pattern and condition on the size and mass production of lipidic PLGA NPs. In the study, organic solutions (acetonitrile containing polymer) in the middle inlet and aqueous phase (lecithin and DSPE-PEG) in the outer inlets generate micro vortices. NPs sizes are affected by flow rates and Reynolds number, as in higher Reynolds numbers $(\mathrm{Re}=150,75)$, NPs size decreases ( $55 \mathrm{~nm}$ and $81 \mathrm{~nm}$, respectively). The authors claimed that their microfluidics has higher productivity (up to $3 \mathrm{~g} \mathrm{~h}^{-1}$ ) which is 1000 -fold of the conventional 2D HF microfluidics. Gdowski et $a .^{88}$ used a micromixer with herringbone pattern (Fig. 6X) to promote the mixing of PLGA and curcumin in acetonitrile with an aqueous phase containing DSPE-PEG. They optimized NPs size to $102.11 \mathrm{~nm}$ with $4.4 \%$ drug loading and $58.8 \%$ encapsulation efficiency. However, in vivo assessment with mice bearing prostate cancer cells (C4-2B) showed that NPs are completely removed from the body after $24 \mathrm{~h}$ (Fig. 6XI).

\section{PLGA-based microparticles}

Microparticles have great importance in biomedical applications because of their capability in the delivery of a broad range of drugs, higher encapsulation efficiency, controlled and stimuli release..$^{25,39,89,90}$ Due to the good properties of PLGA for biomedical applications, researchers have considered different geometries, chips, and configurations to produce PLGA-based microcarriers with tunable size and morphology. Table 2 summarizes PLGA MPs production in various microfluidic systems with different agents to be used in drug delivery systems. The current section covers PLGA MPs produced in microfluidic systems.

Typically, MPs in microfluidics are produced via template droplets that turn into MPs through various reactions or in the case of PLGA, by solvent evaporation or diffusion..$^{25}$ Rapid processing in microfluidics needs a volatile solvent to evaporate rapidly as well as dissolve organic component. For this aim, organic solvents such as DMC, chloroform, and toluene are frequently used to produce droplets in an aqueous continuous phase. The solvent is important since it can affect the production process or even the properties of the final particles. For instance, encapsulation efficiency, an initial burst release of enoxacin (ENX)-loaded PLGA MPs synthesized in a PDMS microfluidics is controlled by changing the solvent from DMC to dichloromethane (DCM) ${ }^{100}$ MPs produced by PLGA dissolved in DMC exhibited higher encapsulation efficiency (56.5\%) and initial burst release (14.8\%) compared with DCM (encapsulation efficiency $15.4 \%$, initial burst release: $12 \%$ ). The concentration of the organic phase is effective in the size of particles. With the increase in PLGA concentration $(1,3$, and $5 \mathrm{wt} \%)$ in DMC, MPs size increases from 140 to $160 \mu \mathrm{m}$.

Stability investigation of PLGA MPs has been carried out by various researchers. ${ }^{101} \mathrm{Tu}$ and Lee investigated the effect of PLGA composition and the $\mathrm{pH}$ of the inner phase of water-inoil-in-water $(\mathrm{W} / \mathrm{O} / \mathrm{W})$ double emulsions. They showed that basic phase in double emulsion PLGA microcapsules enhances 
Table 2 PLGA MPs synthesized in microfluidics

\begin{tabular}{|c|c|c|c|c|c|c|c|}
\hline Microfluidic & Channel size & Chip geometry & Number of core & MPs size $(\mu \mathrm{m})$ & PLGA (L/G) & Agent & Ref. \\
\hline PDMS & $100-200$ & Flow-focus & 1 & $10-50$ & $85: 15$ & & 39 \\
\hline Glass capillary & $\begin{array}{l}\text { Inner tube: } 30-50 \mu \mathrm{m} \\
\text { middle tube: } 100 \mu \mathrm{m}\end{array}$ & Co-flow & $1-3$ & $\sim 210$ & $50: 50$ & Doxorubicin hydrochloride & 43 \\
\hline PDMS & & Flow-focus & 1 & $3-6$ & $50: 50$ & - & 23 \\
\hline PDMS & $\begin{array}{l}\text { Height } 100 \mu \mathrm{m} \text { width } \\
200 \mu \mathrm{m}\end{array}$ & T-junction & 1 & $50-65$ & $75: 25$ & $\begin{array}{l}\text { ac-Rb1 } \\
\left(6^{\prime \prime}-O \text {-acetylginsenoside Rb1) }\right.\end{array}$ & 91 \\
\hline PDMS & & Flow-focus & 1 & $\sim 28$ & $50: 50$ & Tanshinone IIA & 93 \\
\hline Capillary & $700 \mu \mathrm{m} \& 1 \mathrm{~mm}$ & Flow-focus & 1 & $\approx 145$ & $75: 25$ & Dexamethasone + latanoprost & 94 \\
\hline PDMS & Height $20 \mu \mathrm{m}$ width $30 \mu \mathrm{m}$ & Flow-focus & Hollow MPs & $<7$ & $50: 50$ & $\begin{array}{l}\text { Celecoxib (CEL) \& sorafenib } \\
\text { (SFN) }\end{array}$ & 95 \\
\hline Glass capillary & - & Flow-focus & 1 & - & $50: 50$ & Gas & 96 \\
\hline $\begin{array}{l}\text { Fluoroelastomer } \\
\text { SIFEL }\end{array}$ & $100 \mu \mathrm{m}$ & Flow-focus & 1 & $67.0 \pm 1.6$ & $75: 25$ & - & 97 \\
\hline FF nozzle & - & Flow-focusing & 1 & $3-6$ & $50: 50$ & Lidocaine & 99 \\
\hline
\end{tabular}

the surface activity and consequently improve colloidal stability. ${ }^{\mathbf{1 0 2}}$ Furthermore, it has been proven that monodisperse MPs produced in microfluidics have better colloidal stability in aqueous dispersion when compared MPs produced a bulk method that tends to aggregate in storage. ${ }^{23}$

Droplets and emulsions stability is also important in the final characteristics of MPs which can be affected by the chip properties and configuration. For instance, PDMS is hydrophobic and it's difficult to produce droplets with varying hydrophobicity. Some modifications have been proposed and used $^{\mathbf{1 0 3 , 1 0 4}}$ such as immersion in the poly(vinyl alcohol)/glycerol solution to produce PTX loaded poly(L-lactic acid) (PLLA) microspheres. ${ }^{42}$ It is of the utmost importance to consider its characteristics in droplet and emulsion productions. Hydrophilic PDMS changes to hydrophobic in contact with organic solutions such as dichloromethane. To prevent this phenomenon which circumvents droplet and particle formation, $\mathrm{Xu}$ et al. used T-junction flow-focusing geometry. In the configuration, water is in contact with MPs wall which surrenders the dichloromethane phase. Bupivacaine loaded PLGA MPs in the range of $10-50 \mu \mathrm{m}$ size produced by capillary device release drug more slowly and also have significantly smaller initial burst release in comparison with conventionally produced particles. $^{39}$

Various studies have been conducted to investigate the microfluidics capability in the control of MPs shape and morphology. In this regard, PLGA-based MPs fabricated with various shape such as honeycomb ${ }^{\mathbf{1 0 5}-107}$ Golf-ball, ${ }^{\mathbf{1 0 6}}$ snowman microcapsules, ${ }^{108}$ porous microbeads. ${ }^{109}$ Hussein et al. reported that surface texture is easily controllable using polymers with various hydrophobicity in a microfluidics system. ${ }^{62}$ They prepared $\mathrm{PLGA}_{100 \mathrm{k}}$ blend with $\mathrm{PLGA}_{50 \mathrm{k}}-b-\mathrm{PEG}_{5 \mathrm{k}} / \mathrm{PLGA}_{100 \mathrm{k}}$ or $\mathrm{PLGA}_{10 \mathrm{k}}-b-\mathrm{PEG}_{20 \mathrm{k}} / \mathrm{PLGA}_{10 \mathrm{k}}$ and PTX as a solution $\left(10 \mathrm{mg} \mathrm{mL}^{-1}\right)$ in DCM as a dispersed phase in the aqueous phase $\left(5 \mathrm{mg} \mathrm{mL}^{-1}\right.$
PVA) with varying ratios in a glass capillary. Results indicated that surface texture is controllable with the blend ratio and consequently it affects encapsulation efficiency and releases kinetics. For example, with an increase in the PEG content ( 0 to more than $60 \mathrm{wt} \%$ ) particles surface changes from a smooth to bumpy appearance and finally breaks into nanoscale micelles. Moreover, encapsulation efficiency drops with an increase in the PEG content, e.g. encapsulation of PTX is reported about $92 \%$ for neat $\mathrm{PLGA}_{100 \mathrm{k}}(\mathrm{PEG}=0)$, while in the case of neat $\mathrm{PLGA}_{50 \mathrm{k}}-b-\mathrm{PEG}_{5 \mathrm{k}}$ it is about $64 \%$. The authors attributed the results to the fact that higher hydrophobic content of polymer promotes more PTX encapsulation. PEG content also enhances the PTX release, as the highest drug release profile (Fig. 7I) is observed for $80 \%$ of $\mathrm{PLGA}_{50 \mathrm{k}}-b-\mathrm{PEG}_{5 \mathrm{k}}$ and the lowest for neat PLGA $_{100 k}$. On one side, the more the hydrophilic content, the more the water adsorption and on the other side the more the PEG content, the higher the roughness and consequently, the more interfacial area available for water diffusion. All together promote drug release for particles with a higher amount of PEG in the polymer blend.

From another point of view, microfluidics provides advantages to load agents with various properties in polymeric matrixes. It enables inorganic material loading into PLGA MPs and hybrid MPs production. For instances, the cross-junction microfluidic system has been applied to encapsulate CdSe/ ZnS QDs in PLGA MPs with the size of 180 to $550 \mu \mathrm{m}$. PLGA along with CdSe/ZnS QDs $(4 \pm 0.5 \mathrm{~nm})$ in chloroform produces droplets in an aqueous phase containing PVA (1 wt/v\%) and chitosan (0.5 wt/v\%) in a flow focusing microchip. ${ }^{110} \mathrm{~W} / \mathrm{O} / \mathrm{W}$ double emulsion template produced in a capillary microfluidics consisted of an inner aqueous phase containing $10 \mathrm{wt} \%$ PEG with 8-hydroxyl-1,3,6-pyrenetrisulfonic acid trisodium salt (a green dye), and sulforhodamine B (a red dye), middle oil phase containing PEG- $b$-PLA with poly( $N$-isopropylacrylamide) 


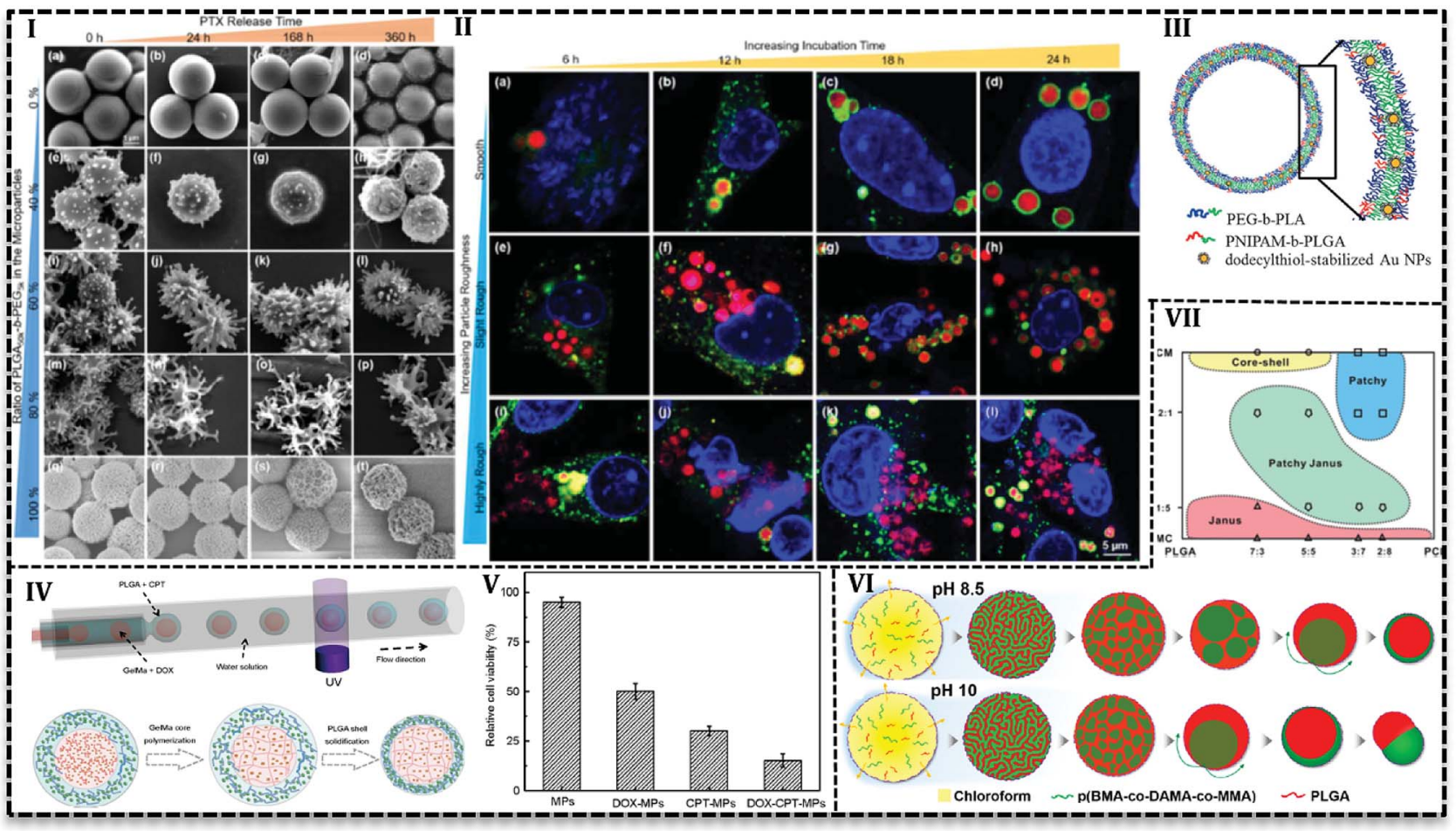

Fig. 7 PLGA-based MPs produced in droplet-based microfluidics. (I) SEM images show the drug release of PLGA $A_{50 k}-b-P E G_{5 k} / P L G A_{100 k}$ polymer MPs with varying polymer ratio in different times. Authors concluded because of diffusion drug release mechanism MPs surface morphology is stable over time. (II) Cell lysosomes were stained with Lyso Tracker Green DND-26 (green) while blue Hoechst was used to label the nuclei. Confocal Laser Scanning Microscope image demonstrates cellular uptake of Nile red labeled MPs with B16-F10 melanoma cell that indicates the MPs are taken up by the cancer cells and the MPs can act as drug carriers to successfully transport hydrophobic drugs to the cancer cells. (III) Schematic of the photo- and thermoresponsive MPs composed of a bilayer of PEG- $b$-PLA and PNIPAM- $b$-PLGA diblock copolymers, containing dodecylthiol-stabilized gold nanoparticles to induce responsive release. (IV) Schematic of W/O/W double emulsion templates generation in a capillary microfluidics and the illustration of the drug-loaded GelMa-PLGA core-shell MPs formation in the device. (V) In vitro analysis of the human colon cancer cell line (HCT116 cells) treated with unloaded MPs, only DOX-loaded MPs, only CPT-loaded MPs, and DOX-CPT-co-loaded MPs for $24 \mathrm{~h}$ revealed that antitumor effect of dual drug loaded MPs is higher than individual drugs. (VI) Schematic of the effect of in the final morphology of the Nile red and coumarin loaded core-shell $\mathrm{pH}$-responsive MPs formation from a homogeneous chloroform drop containing p(BMA-CO-DAMA-CO-MMA) and PLGA in the aqueous phase with $\mathrm{pH} 8.5$ and 10. (VII) Various structures produced by different volume ratio of the solvents (dichloromethane/dimethyl carbonate) and the mass ratio of PLGA/PCL (the total concentration was $40 \mathrm{mg} \mathrm{mL}^{-1}$ ).

(PNIPAM)-b-PLGA (as thermosensitive polymer) and dodecylthiol-stabilized gold NPs (a photosensitive agent) in a mixture of chloroform and hexane, outer aqueous solution containing $10 \mathrm{wt} \%$ PVA. After evaporation, polymersome produced with a double polymer layer (Fig. 7III) in which gold NPs are entrapped in the PNIPAM- $b$-PLGA part. By examination and optimization with various amounts $(2,5$, and $10 \mathrm{wt} \%)$ of PNIPAM- $b$-PLGA, they concluded that $5 \mathrm{wt} \%$ is needed to produce thermosensitive polymersome. On the other hand, a higher amount of thermosensitive polymer results in defects and finally ruptures of the polymersome. The release mechanism of polymersome induced by temperature and laser illumination indicated that in thermoresponsive release pores form in the polymersome and releases the load gradually. However, laser triggered release starts with local hot spots formation which finally results in the rupture of bilayer. ${ }^{111}$ PLGA microspheres containing $\mathrm{TiO}_{2} \mathrm{NPs}$ on the surface produced in FF chip. PLGA and titanium tetraisopropoxide (TTIP) in DCM generated droplets in aqueous solution $90 \mathrm{wt} \%$ of glycerol and
$0.5 \mathrm{wt} \%$ of PVA. $\mathrm{TiO}_{2}$ forms upon the contact with aqueous phase as a result of TTIP hydrolysis also make MPs with a wrinkled surface. With the increase in the mass ratio of TTIP/ PLGA (4/30 to 8/30), surface wrinkles get deeper and after 12/30 particle changes to non-spherical. Tanshinone IIA incorporated into MPs as a model drug with encapsulation efficiency higher than $80 \%$ and results from in vitro drug release showed the deeper the wrinkles on the surface the higher drug release rate. ${ }^{93}$

\subsection{Core-shell microparticles}

Core-shell MPs or microcapsules contain gas, liquid or solid core (or multiple cores) covered up in the shell. ${ }^{25,112}$ These morphologies offer a broad range of advantages such as dual or multiple drug delivery, controlled and prolonged release, protection against active agents. ${ }^{113}$ Moreover, the core-shell structure can deliver chemically active agents and protect them from body proteins, immune system, and degradation. Microchannel-based synthesis offers unique opportunities to 
produce core-shell, multiple cores in one shell and even multiple shells on one core. It is done through solidification of single/multiple emulsions.

For instance, Martín-Banderas et al. encapsulated gentamycin sulfate (GS) in PLGA MPs with one or more core and microcapsules. ${ }^{\mathbf{1 1 4}}$ They used an FF configuration with PLGA and drug solution focused with an aqueous solution to produce MPs. They used the same configuration with multiple concentric needles with air in the inner needle to produce microcapsules. They reported high drug loading (10 to 30\%) and encapsulation (42 to 85\%) efficiencies for the microcapsules compared with MPs. In another report, alginate shell on PLGA MPs synthesized in a flow focusing capillary microfluidic used to control the release of rifampicin in the size range between 15 to $50 \mu \mathrm{m}$. The core-shell morphology produced from $\mathrm{W} / \mathrm{O} / \mathrm{W}$ double emulsion templates in which consisted of inner aqueous phase $(0.5 \%$ sodium alginate and $10 \%(\mathrm{w} / \mathrm{v})$ PVA), middle oil phase (DCM with 20\% PLGA) and outer aqueous phase $\left(10 \%(\mathrm{w} / \mathrm{v})\right.$ PVA and $4 \%$ calcium chloride $\left.\left(\mathrm{CaCl}_{2}\right)\right)$. Both shell and core sizes affect the drug release and by an increase in the MPs size, initial burst release decreases. Moreover, coreshell structure exhibited higher drug content $(\sim 6.4 \%)$ than microspheres $(4.26 \pm 0.54)$ and also higher encapsulation efficiency $(70.47 \pm 1.85 \%)$ compared with microspheres $(46.78 \pm$ $5.89)$ with similar size $(\sim 50 \mu \mathrm{m})$. In agreement with results from other investigations, ${ }^{65}$ the authors attributed the results to shell layer that prevents the drug from diffusion to solvent in the evaporation stage of the fabrication process. ${ }^{\mathbf{1 1 3}, 115}$

PLGA-based MPs containing liquid cores are thermodynamically unstable and shell rupture takes place during solidification and degradation that causes to burst release of the loaded drug. Various strategies have been used to overcome this shortcoming and improve MPs stability. For instance, gelatin methacrylate (GelMa) used as a crosslinking agent to avoid rupture or fusion of cores. ${ }^{43}$ PLGA-based core-shell structure containing DOX hydrochloride and camptothecin (CPT) fabricated by one-step co-flow capillary microfluidics (Fig. 7IV). Double $\mathrm{W} / \mathrm{O} / \mathrm{W}$ emulsion produced in three-concentric capillary tubes. Inner aqueous phase contained hydrophilic drug (DOX hydrochloride) and GelMa $(15 \% \mathrm{w} / \mathrm{v})$ while the outer aqueous phase contains PVA (2\% w/v). Hydrophobic drug (CPT) dissolved in DCM with PLGA polymer as a middle oil solution. Emulsions polymerized by UV light to solidify core to produce stable core and release two or more therapeutics sustainably. They achieved the varying size of MPs and core numbers (one, double and triple cores in one shell) with various orifice size and fluid flow rates. Results illustrated that increase in the shell thickness $(22,40$, and $60 \mu \mathrm{m})$ leads to higher encapsulation efficiency of CPT ( $\sim 46,57$ and $61 \%)$ and DOX hydrochloride (85, 89 and 93\%) and higher drug content (4.06 $\pm 0.02,6.17 \pm 0.15$ and $6.88 \pm 0.24)$. In vitro evaluation with human colon cancer cell line (HCT116) (Fig. 7V) and liver cancer cell line (HepG2) revealed the synergistic antitumor effect of two drugs. Antitumor effect of dual drug loaded MPs (less than 20\% HCT116 cells and $10 \%$ HepG2 cells survive) is higher than individual drugs either DOX or CPT (50\% HCT116 cells and 60\% HepG2 cells are killed). In another approach, Montazeri et al. ${ }^{92}$ improved the PDMS based double flow focusing chip to produce a partially hydrophilic-hydrophobic microfluidic device. They added a surfactant (Silwet L-77@) to the curing agent and prepolymer of the PDMS in chip preparation stage to improve the wettability of the chip. They investigated contact angle of water droplet by the surface of PDMS that led them to choose $0.5 \mathrm{wt} \%$ among the various concentration of surfactant $(0,0.2,0.5$ and $0.8 \mathrm{wt} \%$ ) to produce PLGA based MPs with average $20 \mu \mathrm{m}$. Results reveal that modification enables $\mathrm{H}_{2} \mathrm{O}_{2}$ solution delivery as an oxygen generator into islet transplantation over an extended time up to 30 days.

\subsection{Janus microparticles}

Janus or bifacial MPs have excellent properties such as tunable and controllable asymmetry in shape, composition, the capability to load multiple and even incompatible agents. ${ }^{\mathbf{8 9 , 1 1 6}}$ In addition to these properties, recently Janus MPs production in microfluidics attracted more attention because of their facility and capability in the synthesis and control of physical and chemical features. ${ }^{\mathbf{1 1 6 , 1 1 7}}$

In order to achieve Janus MPs, two-face droplets are necessary which will solidify to MPs after droplet consolidation. Therefore, two miscible fluids form droplets in a third immiscible fluid. Min et al. dissolved PLGA and poly(butyl methacrylate-co-(2-dimethylaminoethyl) methacrylate-co-methyl methacrylate) (p(BMA-co-DAMA-co-MMA)) in an organic solvent (chloroform) as the dispersed phase and the aqueous phase of $10 \mathrm{w} / \mathrm{w}$ PVA and $0.1 \mathrm{M} \mathrm{NaOH}$ as a continuous phase to produce droplets in a glass capillary. ${ }^{118}$ At $\mathrm{pH}$ 8.5, by the diffusion of chloroform into the continuous phase and inversion of polymeric domains within the droplets, PLGA core forms in the $\mathrm{p}$ (BMA-co-DAMA-co-MMA) shell. Nile red (hydrophobic) and coumarin (hydrophilic) model drugs tend to accumulate in PLGA core and p(BMA-co-DAMA-co-MMA) shell, respectively. However, in $\mathrm{pH}$ 10, particles tend to form acorn-shaped Janus MPs (Fig. 7VI). Shell over multiple cores forms when the solvent is replaced with toluene over pH 8.5 to 10 . Since the evaporation of toluene happens very fast (2.4-fold faster than chloroform), coalescence of PLGA domain stay separately during evaporation.

PLGA and poly(3-caprolactone) (PCL) MPs with patchy and Janus structures have been produced in droplet microfluidics in order to control drug release. ${ }^{\mathbf{1 1 9 , 1 2 0}}$ A solution of PLGA and PCL in DMC or DCM produced organic droplets in a continuous aqueous solution of PVA ( $2 \mathrm{wt} \%$ ). The strategy to achieve various morphologies was switching between two organic solvents. Polymer solution in DMC produces MPs with Janus particles (average size $24.42 \mu \mathrm{m}$ ) while in DCM, core-shell (average size $47 \mu \mathrm{m})$ structure with PLGA in the core and smooth surface. ${ }^{119}$ They investigated the effect of solvent concentration and the mass ratio of PLGA : PCL on the final MPs morphology and provided a diagram in order to easily choose the ratio for the desired final product. For example, core-shell MPs generated with a higher portion of PLGA (PLGA : PCL as $7: 3$ or $5: 5$ ) and anisotropic patchy particles harvested in the lower portions (PLGA : PCL as $3: 7$ or $2: 8$ ). At an equal mass ratio of polymers, 
Janus particle and patchy morphology produced with a change in the DCM : DMC volume ratio $1: 5$ and $2: 1$, respectively. ${ }^{\mathbf{1 2 0}}$

In another strategy, Kang et al. ${ }^{117}$ produced PLGA Janus particles in which both parts are PLGA. For that purpose, they used two solvents to produce MPs; ethyl acetate (EA) and silicone oil are good and bad solvents for PLGA, respectively. The $\mathrm{O} / \mathrm{W}$ emulsion in glass capillary which consisted of PLGA dissolved in two solvents as oil phase while continuous PVA aqueous phase as water flow. Faster evaporation of EA compared with removal of silicon oil, in the MPs formation stage produces Janus shape. Researchers claimed that the diameter of each part Janus PLGA MPs is predictable using theoretical and mathematical calculations.

\section{Opportunities and challenges}

PLGA DDSs production has started since two decades ago. It's obvious the method and produced NPs have undergone significant improvements since the first production of PLGA based NPs in microfluidics. PLGA based NPs are being synthesized with advanced features such as targeting ligands, lipid layers, stimuli-responsive, co-loaded with drugs and imaging agents. Various microfluidic chips (e.g. 2D, 3D, arc, and origami) have been used to investigate effective factors in the production process such as flow ratio, mixing time and microchannel size. Droplet-based microfluidics is being employed for the synthesis of complex MPs. This category of microfluidics has offered a powerful platform to control size, size distribution, rapid processing and uploading of drugs with varying hydrophobicity and properties. MPs are being fabricated with core-shell structure and even with multiple cores and/or shell. Apart from the encapsulation of incompatible drugs and/or unstable agents with varying physicochemical properties, the core-shell structure can make it possible to achieve sustained and even sequential release.

Although the technology provides a wide range of opportunities, it is not free from limitations and drawbacks that can hinder its application in large scales.

The first important issue that should be addressed by prospective researches is the fouling of PLGA NPs during the precipitation process and clogging the micro-size channels. As a matter of fact, the microfluidically production of PLGA DDS is considered as a continuous process which avoids batch-tobatch variation. This feature has been arguably accepted as one of the successful aspects of the technology. However, scientists are struggling with a huge number of failed chips in the lab due to the clogging of the microchannels. Not only fouling of NPs but also problems are being reported related to the wettability and hydrophobicity issues in MPs production. Despite all attempts done to solve the problems, results are not admissible and show that microfluidics hardware and choice needs to be selected according to every synthesis process.

Moreover, the contribution of various mixers like Tesla micromixers has been introduced and examined before and/or after focusing section of the synthesis process in continuous microfluidics. Two-stage microfluidically synthesis has been reported to control the core and shell more precisely in the production inorganic NPs. ${ }^{\mathbf{1 2 1 , 1 2 2}}$ Such multi-stage could be advantageous in the production of PLGA-based DDSs with sophisticated features. Moreover, screening and preclinical test of drugs in living cells are crucial steps in drug discovery, direct purification and cell treatment on the microfluidic platform could be considered as additional steps toward the increased efficacy and speed.

Furthermore, manipulation of small volumes of liquids in micro-size has been mentioned as an advantage of these apparatuses; however, it is a double-edged sword and could be a disadvantage at the same time. Low volume of liquids can cause a low throughput of the fabrication process that cannot meet the industrial and large-scale production demand. Although there are few reports of high production rates suitable for preclinical and clinical demands, researchers need to search a solution for this very crucial issue. Future microfluidics should be able to produce a higher amount of drugs without sacrificing accuracy and efficiency.

\section{Conclusion}

PLGA based drug delivery systems are being produced with various fabrication methods and nowadays there are plenty of them approved by FDA and also many ongoing preclinical and clinical types of research to make their way to industry. For this purpose, researchers from all around the world look for new routes to produce DDS with more sophisticated features to promote the production and also delivery efficiency. In this context, we accented various type of microfluidic systems used for the production of PLGA based drug delivery, properties, and applications of PLGA NPs and MPs fabricated by this technology.

All together and in spite of drawbacks, inspiring abilities of the technique hold a great potential to bring and add exciting features to drug delivery systems e.g. controlled release, regulated surface and flexibility, stimuli-responsive and etc. knowledge gained from numerous examples of represented PLGA based drug delivery systems prepared in the microfluidics can help researchers to select proper reactants, microfluidics type and process to fabricate their goal drug delivery system.

\section{Conflicts of interest}

The authors declare that there is no conflict of interest.

\section{References}

1 I. U. Khan, C. A. Serra, N. Anton and T. Vandamme, Microfluidics: a focus on improved cancer targeted drug delivery systems, J. Controlled Release, 2013, 172(3), 10651074.

2 C. X. Zhao, Multiphase flow microfluidics for the production of single or multiple emulsions for drug delivery, Adv. Drug Delivery Rev., 2013, 65(11-12), 14201446.

3 D. Liu, H. Zhang, F. Fontana, J. T. Hirvonen and H. A. Santos, Current developments and applications of 
microfluidic technology toward clinical translation of nanomedicines, Adv. Drug Delivery Rev., 2018, 128, 54-83.

4 M. Mir, N. Ahmed and A. U. Rehman, Recent applications of PLGA based nanostructures in drug delivery, Colloids Surf., B, 2017, 159, 217-231.

5 C. Engineer, J. Parikh and A. Raval, Review on Hydrolytic Degradation Behavior of Biodegradable Polymers from Controlled Drug Delivery System, Trends Biomater. Artif. Organs, 2011, 25(2), 79-85.

6 F. Ramazani, W. Chen, C. F. van Nostrum, G. Storm, F. Kiessling, T. Lammers, W. E. Hennink and R. J. Kok, Strategies for encapsulation of small hydrophilic and amphiphilic drugs in PLGA microspheres: state-of-the-art and challenges, Int. J. Pharm., 2016, 499(1-2), 358-367.

7 R. A. Jain, The manufacturing techniques of various drug loaded biodegradable poly(lactide-co-glycolide) (PLGA) devices, Biomaterials, 2000, 21(23), 2475-2490.

8 F. Y. Han, K. J. Thurecht, A. K. Whittaker and M. T. Smith, Bioerodible PLGA-Based Microparticles for Producing Sustained-Release Drug Formulations and Strategies for Improving Drug Loading, Front. Pharmacol., 2016, 7, 185.

9 F. Danhier, E. Ansorena, J. M. Silva, R. Coco, A. Le Breton and V. Preat, PLGA-based nanoparticles: an overview of biomedical applications, J. Controlled Release, 2012, 161(2), 505-522.

10 J. M. Lu, X. Wang, C. Marin-Muller, H. Wang, P. H. Lin, Q. Yao and C. Chen, Current advances in research and clinical applications of PLGA-based nanotechnology, Expert Rev. Mol. Diagn., 2009, 9(4), 325-341.

11 H. K. Makadia and S. J. Siegel, Poly Lactic-co-Glycolic Acid (PLGA) as Biodegradable Controlled Drug Delivery Carrier, Polymers, 2011, 3(3), 1377-1397.

12 S. Acharya and S. K. Sahoo, PLGA nanoparticles containing various anticancer agents and tumour delivery by EPR effect, Adv. Drug Delivery Rev., 2011, 63(3), 170-183.

13 J. Ma, S. M.-Y. Lee, C. Yi and C.-W. Li, Controllable synthesis of functional nanoparticles by microfluidic platforms for biomedical applications-a review, Lab Chip, 2017, 17(2), 209-226.

14 R. Karnik, F. Gu, P. Basto, C. Cannizzaro, L. Dean, W. KyeiManu, R. Langer and O. C. Farokhzad, Microfluidic platform for controlled synthesis of polymeric nanoparticles, Nano Lett., 2008, 8(9), 2906-2912.

$15 \mathrm{X}$. Li and X. Jiang, Microfluidics for producing poly (lacticco-glycolic acid)-based pharmaceutical nanoparticles, $A d v$. Drug Delivery Rev., 2018, 128, 101-114.

16 A. Pandey, D. S. Jain and S. Chakraborty, Poly Lactic-Co-Glycolic Acid (PLGA) copolymer and its pharmaceutical application, Handbook of polymers for pharmaceutical technologies: processing and applications, 2015, vol. 2, pp. 151-172.

17 G. M. Whitesides, The origins and the future of microfluidics, Nature, 2006, 442(7101), 368-373.

18 F. Fontana, M. P. A. Ferreira, A. Correia, J. Hirvonen and H. A. Santos, Microfluidics as a cutting-edge technique for drug delivery applications, J. Drug Delivery Sci. Technol., 2016, 34, 76-87.
19 R. Riahi, A. Tamayol, S. A. M. Shaegh, A. M. Ghaemmaghami, M. R. Dokmeci and A. Khademhosseini, Microfluidics for advanced drug delivery systems, Curr. Opin. Chem. Eng., 2015, 7, 101-112.

20 S. T. Sanjay, W. Zhou, M. Dou, H. Tavakoli, L. Ma, F. Xu and $\mathrm{X}$. Li, Recent advances of controlled drug delivery using microfluidic platforms, Adv. Drug Delivery Rev., 2017, 128, 3-28.

21 W. J. Guan and Y. Zhang, Application of Microfluidic Technique in Drug Delivery, Nano LIFE, 2016, 6(3-4), 1642009.

22 Y. S. Lin, C. H. Yang, C. Y. Wang, F. R. Chang, K. S. Huang and W. C. Hsieh, An Aluminum Microfluidic Chip Fabrication Using a Convenient Micromilling Process for Fluorescent Poly(DL-lactide-co-glycolide) Microparticle Generation, Sensors, 2012, 12(2), 1455-1467.

23 A. Perez, R. Hernandez, D. Velasco, D. Voicu and C. Mijangos, Poly (lactic-co-glycolic acid) particles prepared by microfluidics and conventional methods. Modulated particle size and rheology, J. Colloid Interface Sci., 2015, 441, 90-97.

24 P. A. Zhu and L. Q. Wang, Passive and active droplet generation with microfluidics: a review, Lab Chip, 2017, 17(1), 34-75.

25 L. Shang, Y. Cheng and Y. Zhao, Emerging Droplet Microfluidics, Chem. Rev., 2017, 117(12), 7964-8040.

26 B. F. B. Silva, C. Rodriguez-Abreu and N. Vilanova, Recent advances in multiple emulsions and their application as templates, Curr. Opin. Colloid Interface Sci., 2016, 25, 98108.

27 D. Liu, H. Zhang, F. Fontana, J. T. Hirvonen and H. A. Santos, Microfluidic-assisted fabrication of carriers for controlled drug delivery, Lab Chip, 2017, 17(11), 18561883.

28 Z. X. Luo, G. Zhao, F. Panhwar, M. F. Akbar and Z. Q. Shu, Well-designed microcapsules fabricated using dropletbased microfluidic technique for controlled drug release, J. Drug Delivery Sci. Technol., 2017, 39, 379-384.

29 M. A. Holgado, M. J. Cozar-Bernal, S. Salas, J. L. Arias, J. Alvarez-Fuentes and M. Fernandez-Arevalo, Proteinloaded PLGA microparticles engineered by flow focusing: physicochemical characterization and protein detection by reversed-phase HPLC, Int. J. Pharm., 2009, 380(1-2), 147-154.

30 S. Ding, N. Anton, T. F. Vandamme and C. A. Serra, Microfluidic nanoprecipitation systems for preparing pure drug or polymeric drug loaded nanoparticles: an overview, Expert Opin. Drug Delivery, 2016, 13(10), 1447-1460.

31 M. Q. Lu, A. Ozcelik, C. L. Grigsby, Y. H. Zhao, F. Guo, K. W. Leong and T. J. Huang, Microfluidic hydrodynamic focusing for synthesis of nanomaterials, Nano Today, 2016, 11(6), 778-792.

32 A. Abou-Hassan, R. Bazzi and V. Cabuil, Multistep Continuous-Flow Microsynthesis of Magnetic and Fluorescent $\gamma-\mathrm{Fe}_{2} \mathrm{O}_{3} @ \mathrm{SiO}_{2}$ Core/Shell Nanoparticles, Angew. Chem., Int. Ed., 2009, 48(39), 7180-7183. 
33 A. Abou-Hassan, S. Neveu, V. Dupuis and V. Cabuil, Synthesis of cobalt ferrite nanoparticles in continuousflow microreactors, RSC Adv., 2012, 2(30), 11263-11266.

34 M. Takagi, T. Maki, M. Miyahara and K. Mae, Production of titania nanoparticles by using a new microreactor assembled with same axle dual pipe, Chem. Eng. J., 2004, 101(1-3), 269-276.

35 M. Rhee, P. M. Valencia, M. I. Rodriguez, R. Langer, O. C. Farokhzad and R. Karnik, Synthesis of size-tunable polymeric nanoparticles enabled by 3D hydrodynamic flow focusing in single-layer microchannels, Adv. Mater., 2011, 23(12), H79-H83.

36 J. M. Lim, N. Bertrand, P. M. Valencia, M. Rhee, R. Langer, S. Jon, O. C. Farokhzad and R. Karnik, Parallel microfluidic synthesis of size-tunable polymeric nanoparticles using 3D flow focusing towards in vivo study, Nanomedicine, 2014, 10(2), 401-409.

37 V. Genot, S. Desportes, C. Croushore, J. P. Lefevre, R. B. Pansu, J. A. Delaire and P. R. von Rohr, Synthesis of organic nanoparticles in a $3 \mathrm{D}$ flow focusing microreactor, Chem. Eng. J., 2010, 161(1-2), 234-239.

38 L. L. Li, X. D. Li and H. Wang, Microfluidic Synthesis of Nanomaterials for Biomedical Applications, Small Methods, 2017, 1(8), 1700140.

39 Q. Xu, M. Hashimoto, T. T. Dang, T. Hoare, D. S. Kohane, G. M. Whitesides, R. Langer and D. G. Anderson, Preparation of monodisperse biodegradable polymer microparticles using a microfluidic flow-focusing device for controlled drug delivery, Small, 2009, 5(13), 1575-1581.

40 E. Jafarifar, M. Hajialyani, M. Akbari, M. Rahimi, Y. Shokoohinia and A. Fattahi, Preparation of a reproducible long-acting formulation of risperidoneloaded PLGA microspheres using microfluidic method, Pharm. Dev. Technol., 2017, 22(6), 836-843.

41 K. Kinoshita, E. Parra, A. Hussein, A. Utoft, P. Walke, R. de Bruijn and D. Needham, From Single Microparticles to Microfluidic Emulsification: Fundamental Properties (Solubility, Density, Phase Separation) from Micropipette Manipulation of Solvent, Drug and Polymer Microspheres, Processes, 2016, 4(4), 49.

42 T. X. He, Q. L. Liang, K. Zhang, X. Mu, T. T. Luo, Y. M. Wang and G. A. Luo, A modified microfluidic chip for fabrication of paclitaxel-loaded poly(l-lactic acid) microspheres, Microfluid. Nanofluid., 2011, 10(6), 1289-1298.

43 Y. N. Li, D. Yan, F. F. Fu, Y. X. Liu, B. Zhang, J. Wang, L. R. Shang, Z. Gu and Y. Zhao, Composite core-shell microparticles from microfluidics for synergistic drug delivery, Sci. China Mater., 2017, 60(6), 543-553.

44 C. H. Yang, K. S. Huang, A. M. Grumezescu, C. Y. Wang, S. C. Tzeng, S. Y. Chen, Y. H. Lin and Y. S. Lin, Synthesis of uniform poly (d, l-lactide) and poly (d, l-lactide-co-glycolide) microspheres using a microfluidic chip for comparison, Electrophoresis, 2014, 35(2-3), 316322.

45 K. Keohane, D. Brennan, P. Galvin and B. T. Griffin, Silicon microfluidic flow focusing devices for the production of size-controlled PLGA based drug loaded microparticles, Int. J. Pharm., 2014, 467(1-2), 60-69.

46 C. P. Foley, N. Nishimura, K. B. Neeves, C. B. Schaffer and W. L. Olbricht, Flexible microfluidic devices supported by biodegradable insertion scaffolds for convectionenhanced neural drug delivery, Biomed. Microdevices, 2009, 11(4), 915.

47 J. Wang, C. J. Bettinger, R. S. Langer and J. T. Borenstein, Biodegradable microfluidic scaffolds for tissue engineering from amino alcohol-based poly (ester amide) elastomers, Organogenesis, 2010, 6(4), 212-216.

48 C. M. Hwang, A. Khademhosseini, Y. Park, K. Sun and S. H. Lee, Microfluidic chip-based fabrication of PLGA microfiber scaffolds for tissue engineering, Langmuir, 2008, 24(13), 6845-6851.

49 C. M. Hwang, Y. Park, J. Y. Park, K. Lee, K. Sun, A. Khademhosseini and S. H. Lee, Controlled cellular orientation on PLGA microfibers with defined diameters, Biomed. Microdevices, 2009, 11(4), 739-746.

50 J. Cheng, Y. Jun, J. Qin and S. H. Lee, Electrospinning versus microfluidic spinning of functional fibers for biomedical applications, Biomaterials, 2017, 114, 121-143.

51 K. Kumar, A. M. Nightingale, S. H. Krishnadasan, N. Kamaly, M. Wylenzinska-Arridge, K. Zeissler, W. R. Branford, E. Ware, A. J. deMello and J. C. deMello, Direct synthesis of dextran-coated superparamagnetic iron oxide nanoparticles in a capillary-based droplet reactor, J. Mater. Chem., 2012, 22(11), 4704-4708.

52 D. V. R. Kumar, M. Kasture, A. A. Prabhune, C. V. Ramana, B. L. V. Prasad and A. A. Kulkarni, Continuous flow synthesis of functionalized silver nanoparticles using bifunctional biosurfactants, Green Chem., 2010, 12(4), 609-615.

53 S. Duraiswamy and S. A. Khan, Droplet-based microfluidic synthesis of anisotropic metal nanocrystals, Small, 2009, 5(24), 2828-2834.

54 S. E. Lohse, J. R. Eller, S. T. Sivapalan, M. R. Plews and C. J. Murphy, A simple millifluidic benchtop reactor system for the high-throughput synthesis and functionalization of gold nanoparticles with different sizes and shapes, ACS Nano, 2013, 7(5), 4135-4150.

55 A. M. Nightingale, S. H. Krishnadasan, D. Berhanu, X. Niu, C. Drury, R. McIntyre, E. Valsami-Jones and J. C. deMello, A stable droplet reactor for high temperature nanocrystal synthesis, Lab Chip, 2011, 11(7), 1221-1227.

56 A. M. Nightingale, T. W. Phillips, J. H. Bannock and J. C. de Mello, Controlled multistep synthesis in a three-phase droplet reactor, Nat. Commun., 2014, 5, 3777.

57 C. X. Zhao, L. Z. He, S. Z. Qiao and A. P. J. Middelberg, Nanoparticle synthesis in microreactors, Chem. Eng. Sci., 2011, 66(7), 1463-1479.

58 L. H. Hung, S. Y. Teh, J. Jester and A. P. Lee, PLGA micro/ nanosphere synthesis by droplet microfluidic solvent evaporation and extraction approaches, Lab Chip, 2010, 10(14), 1820-1825. 
59 H. Xie, Z. G. She, S. Wang, G. Sharma and J. W. Smith, Onestep fabrication of polymeric Janus nanoparticles for drug delivery, Langmuir, 2012, 28(9), 4459-4463.

60 P. M. Valencia, E. M. Pridgen, B. Perea, S. Gadde, C. Sweeney, P. W. Kantoff, N. H. Bander, S. J. Lippard, R. Langer and R. Karnik, Synergistic cytotoxicity of irinotecan and cisplatin in dual-drug targeted polymeric nanoparticles, Nanomedicine, 2013, 8(5), 687-698.

61 P. M. Valencia, E. M. Pridgen, M. Rhee, R. Langer, O. C. Farokhzad and R. Karnik, Microfluidic platform for combinatorial synthesis and optimization of targeted nanoparticles for cancer therapy, ACS Nano, 2013, 7(12), 10671-10680.

62 M. Hussain, J. Xie, Z. Hou, K. Shezad, J. Xu, K. Wang, Y. Gao, L. Shen and J. Zhu, Regulation of Drug Release by Tuning Surface Textures of Biodegradable Polymer Microparticles, ACS Appl. Mater. Interfaces, 2017, 9(16), 14391-14400.

63 D. Liu, S. Cito, Y. Zhang, C. F. Wang, T. M. Sikanen and H. A. Santos, A versatile and robust microfluidic platform toward high throughput synthesis of homogeneous nanoparticles with tunable properties, Adv. Mater., 2015, 27(14), 2298-2304.

$64 \mathrm{~W}$. Huang and C. Zhang, Tuning the Size of Poly (lactic-co-glycolic Acid)(PLGA) Nanoparticles Fabricated by Nanoprecipitation, Biotechnol. J., 2018, 13(1), 1700203.

65 J. Xu, S. S. Zhang, A. Machado, S. Lecommandoux, O. Sandre, F. Gu and A. Colin, Controllable Microfluidic Production of Drug-Loaded PLGA Nanoparticles Using Partially Water-Miscible Mixed Solvent Microdroplets as a Precursor, Sci. Rep., 2017, 7(1), 4794.

66 S. Libi, B. Calenic, C. E. Astete, C. Kumar and C. M. Sabliov, Investigation on hemolytic effect of poly(lactic co-glycolic) acid nanoparticles synthesized using continuous flow and batch processes, Nanotechnol. Rev., 2017, 6(2), 209-220.

67 M. M. Hasani-Sadrabadi, E. Dashtimoghadam, G. Bahlakeh, F. S. Majedi, H. Keshvari, J. J. Van Dersarl, A. Bertsch, A. Panahifar, P. Renaud, L. Tayebi, M. Mahmoudi and K. I. Jacob, On-chip synthesis of finetuned bone-seeking hybrid nanoparticles, Nanomedicine, 2015, 10(23), 3431-3449.

68 M. H. M. Leung and A. Q. Shen, Microfluidic Assisted Nanoprecipitation of PLGA Nanoparticles for Curcumin Delivery to Leukemia Jurkat Cells, Langmuir, 2018, 34(13), 3961-3970.

69 J. Sun, Y. Xianyu, M. Li, W. Liu, L. Zhang, D. Liu, C. Liu, G. $\mathrm{Hu}$ and $\mathrm{X}$. Jiang, A microfluidic origami chip for synthesis of functionalized polymeric nanoparticles, Nanoscale, 2013, 5(12), 5262-5265.

70 D. Liu, H. Zhang, S. Cito, J. Fan, E. Makila, J. Salonen, J. Hirvonen, T. M. Sikanen, D. A. Weitz and H. A. Santos, Core/Shell Nanocomposites Produced by Superfast Sequential Microfluidic Nanoprecipitation, Nano Lett., 2017, 17(2), 606-614.

71 L. Chronopoulou, C. Sparago and C. Palocci, A modular microfluidic platform for the synthesis of biopolymeric nanoparticles entrapping organic actives, J. Nanopart. Res., 2014, 16(11), 2703.

72 M. Bramosanti, L. Chronopoulou, F. Grillo, A. Valletta and

C. Palocci, Microfluidic-assisted nanoprecipitation of antiviral-loaded polymeric nanoparticles, Colloids Surf., A, 2017, 532, 369-376.

73 K. Zhang, X. Tang, J. Zhang, W. Lu, X. Lin, Y. Zhang, B. Tian, H. Yang and H. He, PEG-PLGA copolymers: their structure and structure-influenced drug delivery applications, $J$. Controlled Release, 2014, 183, 77-86.

74 N. Kolishetti, S. Dhar, P. M. Valencia, L. Q. Lin, R. Karnik, S. J. Lippard, R. Langer and O. C. Farokhzad, Engineering of self-assembled nanoparticle platform for precisely controlled combination drug therapy, Proc. Natl. Acad. Sci. U. S. A., 2010, 107(42), 17939-17944.

75 Q. Feng, J. Liu, X. Li, Q. Chen, J. Sun, X. Shi, B. Ding, H. Yu, Y. Li and X. Jiang, One-Step Microfluidic Synthesis of Nanocomplex with Tunable Rigidity and Acid-Switchable Surface Charge for Overcoming Drug Resistance, Small, 2017, 13(9), 1603109.

76 X. J. Kang, C. X. Luo, Q. Wei, C. Y. Xiong, Q. Chen, Y. Chen and Q. Ouyang, Mass production of highly monodisperse polymeric nanoparticles by parallel flow focusing system, Microfluid. Nanofluid., 2013, 15(3), 337-345.

77 K.-I. Min, H.-J. Lee and D.-P. Kim, Three-dimensional flash flow microreactor for scale-up production of monodisperse PEG-PLGA nanoparticles, Lab Chip, 2014, 14(20), 39873992.

78 J. M. Lim, A. Swami, L. M. Gilson, S. Chopra, S. Choi, J. Wu, R. Langer, R. Karnik and O. C. Farokhzad, Ultra-High Throughput Synthesis of Nanoparticles with Homogeneous Size Distribution Using a Coaxial Turbulent Jet Mixer, ACS Nano, 2014, 8(6), 6056-6065.

79 R. J. Bose, S. H. Lee and H. Park, Lipid-based surface engineering of PLGA nanoparticles for drug and gene delivery applications, Biomater. Res., 2016, 20(1), 34.

80 A. Armada-Moreira, E. Taipaleenmaki, F. Itel, Y. Zhang and B. Stadler, Droplet-microfluidics towards the assembly of advanced building blocks in cell mimicry, Nanoscale, 2016, 8(47), 19510-19522.

81 Q. Feng, L. Zhang, C. Liu, X. Li, G. Hu, J. Sun and X. Jiang, Microfluidic based high throughput synthesis of lipidpolymer hybrid nanoparticles with tunable diameters, Biomicrofluidics, 2015, 9(5), 052604.

82 W. Bao, R. Liu, Y. Wang, F. Wang, G. Xia, H. Zhang, X. Li, H. Yin and B. Chen, PLGA-PLL-PEG-Tf-based targeted nanoparticles drug delivery system enhance antitumor efficacy via intrinsic apoptosis pathway, Int. J. Nanomed., 2015, 10, 557.

83 J. X. Jiang, H. W. Chen, C. Yu, Y. Y. Zhang, M. Y. Chen, S. Tian and C. Y. Sun, The promotion of salinomycin delivery to hepatocellular carcinoma cells through EGFR and CD133 aptamers conjugation by PLGA nanoparticles, Nanomedicine, 2015, 10(12), 1863-1879.

84 L. Zhang, Q. Feng, J. Wang, J. Sun, X. Shi and X. Jiang, Microfluidic synthesis of rigid nanovesicles for 
hydrophilic reagents delivery, Angew. Chem., Int. Ed. Engl., 2015, 54(13), 3952-3956.

85 P. M. Valencia, P. A. Basto, L. Zhang, M. Rhee, R. Langer, O. C. Farokhzad and R. Karnik, Single-step assembly of homogenous lipid-polymeric and lipid-quantum dot nanoparticles enabled by microfluidic rapid mixing, ACS Nano, 2010, 4(3), 1671-1679.

86 A. J. Mieszawska, Y. Kim, A. Gianella, I. van Rooy, B. Priem, M. P. Labarre, C. Ozcan, D. P. Cormode, A. Petrov and R. Langer, Synthesis of polymer-lipid nanoparticles for image-guided delivery of dual modality therapy, Bioconjugate Chem., 2013, 24(9), 1429-1434.

87 Y. Kim, B. Lee Chung, M. Ma, W. J. Mulder, Z. A. Fayad, O. C. Farokhzad and R. Langer, Mass production and size control of lipid-polymer hybrid nanoparticles through controlled microvortices, Nano Lett., 2012, 12(7), 35873591.

88 A. Gdowski, K. Johnson, S. Shah, I. Gryczynski, J. Vishwanatha and A. Ranjan, Optimization and scale up of microfluidic nanolipomer production method for preclinical and potential clinical trials, J. Nanobiotechnol., 2018, 16(1), 12.

89 E. E. Ekanem, Z. Zhang and G. T. Vladisavljevic, Facile Production of Biodegradable Bipolymer Patchy and Patchy Janus Particles with Controlled Morphology by Microfluidic Routes, Langmuir, 2017, 33(34), 8476-8482.

90 M. J. Cozar-Bernal, M. A. Holgado, J. L. Arias, I. MunozRubio, L. Martin-Banderas, J. Alvarez-Fuentes and M. Fernandez-Arevalo, Insulin-loaded PLGA microparticles: flow focusing versus double emulsion/ solvent evaporation, J. Microencapsulation, 2011, 28(5), 430-441.

91 R. Samimi, M. Salarian, W. Z. Xu, E. M. Lui and P. A. Charpentier, Encapsulation of Acetyl Ginsenoside Rb1 within Monodisperse Poly (dl-lactide-co-glycolide) Microspheres Using a Microfluidic Device, Ind. Eng. Chem. Res., 2014, 53(28), 11333-11344.

92 L. Montazeri, S. Bonakdar, M. Taghipour, P. Renaud and H. Baharvand, Modification of PDMS to fabricate PLGA microparticles by a double emulsion method in a single microfluidic device, Lab Chip, 2016, 16(14), 2596-2600.

93 H. Dong, G. Tang, T. Ma and X. Cao, One-step fabrication of inorganic/organic hybrid microspheres with tunable surface texture for controlled drug release application, $J$. Mater. Sci.: Mater. Med., 2016, 27(1), 7.

94 R. A. Leon, A. Somasundar, A. Z. M. Badruddoza and S. A. Khan, Microfluidic Fabrication of Multi-Drug-Loaded Polymeric Microparticles for Topical Glaucoma Therapy, Part. Part. Syst. Charact., 2015, 32(5), 567-572.

95 R. Vasiliauskas, D. Liu, S. Cito, H. Zhang, M. A. Shahbazi, T. Sikanen, L. Mazutis and H. A. Santos, Simple Microfluidic Approach to Fabricate Monodisperse Hollow Microparticles for Multidrug Delivery, ACS Appl. Mater. Interfaces, 2015, 7(27), 14822-14832.

96 W. J. Duncanson, M. Zieringer, O. Wagner, J. N. Wilking, A. Abbaspourrad, R. Haag and D. A. Weitz, Microfluidic synthesis of monodisperse porous microspheres with sizetunable pores, Soft Matter, 2012, 8(41), 10636-10640.

97 I. Morita, Y. Ando and Y. J. Heo, Microsphere formation using SIFEL microfluidic devices with organic-solvent resistance, J. Adv. Mech. Des. Syst., 2017, 11(2), JAMDSM0031.

98 T. Schneider, G. H. Chapman and U. O. Hafeli, Effects of chemical and physical parameters in the generation of microspheres by hydrodynamic flow focusing, Colloids Surf., B, 2011, 87(2), 361-368.

99 M. A. Holgado, J. L. Arias, M. J. Cozar, J. Alvarez-Fuentes, A. M. Ganan-Calvo and M. Fernandez-Arevalo, Synthesis of lidocaine-loaded PLGA microparticles by flow focusing. Effects on drug loading and release properties, Int. J. Pharm., 2008, 358(1-2), 27-35.

100 H. G. Kim, K. M. Kim, Y. H. Kim, S. H. Lee and G. M. Kim, Preparation of Monodisperse ENX-Loaded PLGA Microspheres Using a Microfluidic Flow-Focusing Device, J. Biobased Mater. Bioenergy, 2013, 7(1), 108-114.

101 T. T. Kong, Z. Liu, Y. Song, L. Q. Wang and H. C. Shum, Engineering polymeric composite particles by emulsiontemplating: thermodynamics versus kinetics, Soft Matter, 2013, 9(41), 9780-9784.

$102 \mathrm{~F}$. Tu and D. Lee, Controlling the stability and size of double-emulsion-templated poly(lactic-co-glycolic) acid microcapsules, Langmuir, 2012, 28(26), 9944-9952.

103 Q. Tu, J. C. Wang, Y. R. Zhang, R. Liu, W. M. Liu, L. Ren, S. F. Shen, J. Xu, L. Zhao and J. Y. Wang, Surface modification of poly(dimethylsiloxane) and its applications in microfluidics-based biological analysis, Rev. Anal. Chem., 2012, 31(3-4), 177-192.

104 W. Qiu, C. Wu and Z. Wu, Surface Modification of PDMS in Microfluidic Devices, Concise Encyclopedia of High Performance Silicones, 2014, pp. 141-150.

105 J. Ma, Y. S. Hui, M. Zhang, Y. Yu, W. Wen and J. Qin, Facile synthesis of biomimetic honeycomb material with biological functionality, Small, 2013, 9(4), 497-503.

106 J. H. Lee, C. S. Lee and K. Y. Cho, Enhanced cell adhesion to the dimpled surfaces of golf-ball-shaped microparticles, ACS Appl. Mater. Interfaces, 2014, 6(19), 16493-16497.

107 M. Gultekinoglu, X. Jiang, C. Bayram, K. Ulubayram and M. Edirisinghe, Honeycomb-like PLGA-b-PEG Structure Creation with T-junction Micro Droplets, Langmuir, 2018, 34(27), 7989-7997.

108 M. H. Lee, K. C. Hribar, T. Brugarolas, N. P. Kamat, J. A. Burdick and D. Lee, Harnessing Interfacial Phenomena to Program the Release Properties of Hollow Microcapsules, Adv. Funct. Mater., 2012, 22(1), 131-138.

109 S. W. Choi, Y. Zhang and Y. N. Xia, Fabrication of Microbeads with a Controllable Hollow Interior and Porous Wall Using a Capillary Fluidic Device, Adv. Funct. Mater., 2009, 19(18), 2943-2949.

110 J. Y. Chang, C. H. Yang and K. S. Huang, Microfluidic assisted preparation of $\mathrm{CdSe} / \mathrm{ZnS}$ nanocrystals encapsulated into poly(DL-lactide-co-glycolide) microcapsules, Nanotechnology, 2007, 18(30), 305305. 
111 E. Amstad, S. H. Kim and D. A. Weitz, Photo- and thermoresponsive polymersomes for triggered release, Angew. Chem., Int. Ed. Engl., 2012, 51(50), 12499-12503.

112 S. W. Kim, K. H. Hwangbo, J. H. Lee and K. Y. Cho, Microfluidic fabrication of microparticles with multiple structures from a biodegradable polymer blend, RSC Adv., 2014, 4(87), 46536-46540.

113 J. Wu, T. Kong, K. W. K. Yeung, H. C. Shum, K. M. C. Cheung, L. Wang and M. K. T. To, Fabrication and characterization of monodisperse PLGA-alginate core-shell microspheres with monodisperse size and homogeneous shells for controlled drug release, Acta Biomater., 2013, 9(7), 7410-7419.

114 L. Martín-Banderas, M. Flores-Mosquera, P. Riesco-Chueca, A. Rodríguez-Gil, Á. Cebolla, S. Chávez and A. M. Gañán-Calvo, Flow focusing: a versatile technology to produce size-controlled and specific-morphology microparticles, Small, 2005, 1(7), 688-692.

115 T. Kong, J. Wu, K. W. Yeung, M. K. To, H. C. Shum and L. Wang, Microfluidic fabrication of polymeric core-shell microspheres for controlled release applications, Biomicrofluidics, 2013, 7(4), 44128.

116 T. Nisisako, Recent advances in microfluidic production of Janus droplets and particles, Curr. Opin. Colloid Interface Sci., 2016, 25, 1-12.
117 Z. X. Kang, T. T. Kong, L. Y. Lei, P. G. Zhu, X. W. Tian and L. Q. Wang, Engineering particle morphology with microfluidic droplets, J. Micromech. Microeng., 2016, 26(7), 075011.

118 N. G. Min, M. Ku, J. Yang and S. H. Kim, Microfluidic Production of Uniform Microcarriers with Multicompartments through Phase Separation in Emulsion Drops, Chem. Mater., 2016, 28(5), 1430-1438.

119 W. X. Li, H. Dong, G. N. Tang, T. Ma and X. D. Cao, Controllable microfluidic fabrication of Janus and microcapsule particles for drug delivery applications, $R S C$ Adv., 2015, 5(30), 23181-23188.

120 X. Cao, W. Li, T. Ma and H. Dong, One-step fabrication of polymeric hybrid particles with core-shell, patchy, patchy Janus and Janus architectures via a microfluidic-assisted phase separation process, RSC Adv., 2015, 5(97), 7996979975.

121 R. Gao, N. Choi, S.-I. Chang, E. K. Lee and J. Choo, Realtime analysis of diaquat dibromide monohydrate in water with a SERS-based integrated microdroplet sensor, Nanoscale, 2014, 6(15), 8781-8786.

122 A. Yashina, I. Lignos, S. Stavrakis and J. Choo, Scalable production of CuInS $2 / \mathrm{ZnS}$ quantum dots in a two-step droplet-based microfluidic platform, J. Mater. Chem. C, 2016, 4(26), 6401-6408. 